LOCKHEED MATINA

\author{
RECEIVED \\ HOV 211995 \\ QSATI

\section{Adjustable Speed Drives: Applications and R\&D Needs}

\author{
Victor R. Stefanovic \\ Consultant
}

August 1994

MANAGED BY

LOCKHEED MARTIN ENERGY SYSTEMS, INC.

FOR THE UNITED STATES

DEPARTMENT OF ENERGY

UCN-13675 (6 6-05) 
This report has ben reproduced directly from the best available copy.

Available to DOE and DOE contractors from the Office of Scientific and Technical information, P.O. Box 62, Oak Ridge, TN 37831; prices available from (615) 576-8401, FTS 626-8401.

Available to the public from the National Technical Information Service, U.S. Department of Commerce, 5285 Port Royal Rd., Springfield, VA 22161.

This report was propared as an account of work sponsored by an agency of the United States Government. Neither the United States Government nor any agency thereof, nor any of their employees, makes any warranty, express or implied, or assumes any legal liability or responsibility for the accuracy. completeness, or usefuiness of any information, apparatus, product, or process disclosed, or represents that its use would not infringe privately owned rights. Reference herein to any specific commercial product, process, or service by trade name, trademark, manufacturer, or otherwise, does not necessarily constitute or imply its endorsement, recommendation, or favoring by the United States Government or any agency thereof. The views and opinions of authors expressed herein do not necessarily state or reflect those of the United States Government or any agency thereof. 


\section{Adjustable Speed Drives:}

Applications and R\&D Needs

Received:

August 1994

Published:

September 1995

\section{Prepared by:}

Victor R. Stefanovic

Consultant

for

Oak Ridge National Laboratory

P.O. Box 2008

Oak Ridge, Tennessee 37831

managed by

Lockheed Martin Energy Systems, Inc.

for the

U.S. Department of Energy

Contract DE-AC05-840R21400 


\section{TABLE OF CONTENTS}

\section{Page Number}

EXECUTIVE SUMMARY

2.1 Low Voltage ASDs

2.2 Medium Voltage ASDs

2.2.1 Voltage Source ASDs 11

2.2.2 Current Source ASDs 14

2.2.2.1 Forced Commutated Inverters 15

2.2.2.2 Load Commutated Inverters 19

2.3 ASD Control

19

2.3.1 Constant Volts/Hz Control

21

2.3.2 Field Oriented (Vector) Control 21

2.4 ASD Technical Issues 26

2.4.1 Harmonics Injected Into Power Grid 26

2.4.2 ASD Sensitivity To Power Quality Problems 30

2.4.3 ASD Output Harmonics

31

2.4.4 Motor Insulation Stress

31

2.5 Technical Summary 
3.1 Applications With Mandatory Speed Control 36

3.1.1 Process Improvements 37

3.1.2 Product Improvements 38

3.2 Applications Where Speed Control Is Optional 40

3.3 Emerging Applications 44

3.3.1 Automotive Applications 45

3.3.2 Household Applications 47

4. IMPEDIMENTS TO ASD MARKET PENETRATION 50

4.1 Applications With Mandatory Speed Control 51 -Impediment Factors

4.1.1 Technical Impediments 51

4.1.2 Application Impediments 53

4.1.3 Procurement Impediments 55

4.1.4 Economic Impediments 55

4.1.5 General Impediments 55

4.2 Applications With Optional Speed Control 56 -Impediment Factors

4.2.1 Technical Impediments 56

4.2.2 Application Impediments 57

4.2.3 Procurement Impediments 58

4.2.4 Economic Impediments 59

4.2.5 General Impediments . 59

4.3 Emerging Applications $\quad 60$ -Impediment Factors 
5.1 Short Term Benefits $\quad 62$

5.1 .1 Cost Reduction 62

5.1.2 Reliability Improvements 66

5.1.3 Performance Improvements 67

5.1.4 User Education And General Factors 68

5.2 Long Term Benefits 69

5.2.1 Development Of New Power Switches 69

5.2.2 Application Of Superconductivity to ASDs 70

6. CONCLUSION

71

REFERENCES

73 


\section{LIST OF FIGURES}

FIGURE

$\underline{\text { PAGE }}$

1: GENERAL BLOCK DIAGRAM OF A TYPICAL 4 INDUSTRIAL ADJUSTABLE SPEED DRIVE

2: INDUSTRIAL AC ADJUSTABLE SPEED DRIVES 6

3: CIRCUIT DIAGRAM OF A TYPICAL LOW VOLTAGE, 7 GENERAL PURPOSE, PWM CONTROLLED ASD

4: THREE-PHASE PWM VOLTAGE CONTROL

5: SIX-STEP VOLTAGE WAVE FORMS

6: EXAMPLE OF OUTPUT VOLTAGES OF A

THREE-LEVEL PWM INVERTER

7: CONCEPTUAL SCHEMATIC OF THREE-LEVEL, NEUTRAL POINT CLAMPED (NPC) ASD

8: CURRENT SOURCE ASD, BLOCK DIAGRAM 14

9: AUTO SEQUENTIALLY COMMUTATED

16 CURRENT SOURCE INVERTER (ASCI) 
11: CURRENT SOURCE INVERTER WITH

GTO PWM OUTPUT CONTROL

12: LOAD COMMUTATED INVERTER

13: SEVERAL EXAMPLES OF CONSTANT V/HZ CURVES

14: DIRECT FIELD ORIENTED CONTROL

15: INDIRECT FIELD ORIENTED CONTROL

16: ASD WITH DIODE RECTIFIER - INPUT CURRENT

17: 12-PULSE CONFIGURATION OF AN ASD INPUT RECTIFIER

18: ASD INPUT CURRENT WITH 6 AND 12-PULSE INPUT RECTIFIER 


\section{EXECUTIVE SUMMARY}

The largest opportunity for the growth of adjustable speed drives (ASDs) during the next 5-6 years is in pump, fan and compressor (PFC) applications where a constant, fixed speed operation is converted to adjustable speed in order to realize energy savings. Inverter supplied induction motors are and will continue to be predominately used in these applications.

Over the long term (10-15 years), the greatest ASD growth is expected in large volume consumer applications: first in hybrid electric vehicles (EVs) and, a little later, in residential heating, ventilation and air-conditioning (HVAC). Both induction and a variety of AC Permanent Magnet motors are expected to be the dominant technology in this new field.

The traditional ASD applications in industries which require adjustable speed (such as machine tools, robotics, steel rolling, extruders, paper mill finishing lines, etc.) offer a relatively limited potential for above average ASD growth since most of these applications have already converted to electronic speed control. As a result, ASD growth in this sector will essentially track the growth of the corresponding industries.

If realized, both short and long term ASD growth opportunities will result in significant advancements of ASD technology, which will then substantially affect all other, more fragmented, ASD applications. In fact, any single large volume ASD application will serve as a catalyst for improving ASD characteristics in all other ASD applications with the same voltage rating.

ASD cost and reliability (defined in the context of application compatibility) are the two most important factors which will determine whether the ASD growth opportunities are realized. Conversely, any technological improvement which carries a cost increase will be restricted to niche applications, at best. Consequently, future $R$ \& $D$ efforts should be directed to secure reduction in ASD cost and improvement in ASD reliability. A specific action plan is outlined in this report. 


\section{INTRODUCTION}

This report has the following objectives:

1. To identify key opportunities for the application of Adjustable Speed Drives, (ASDs).

2. To characterize these applications in terms of ASD requirements.

3. To examine and analyze the environment in which ASD applications currently take place and to assess the present ASD technology with respect to key applications.

4. To identify the obstacles and impediments to increased ASD market penetration.

5. To identify an action plan which, if implemented, would result in a higher than normal rate of ASD application growth over the next 5-15 years.

The approach taken in addressing these objectives is to consider all major factors which influence ASD market penetration:

- Technology (reliability and performance)

- Ease of application (common efficiency standards and communication protocols, application knowledge and system issues)

- Economic issues

-ASD cost structure

-Sensitivity of market penetration to ASD price

-Cost of compliance with new harmonic distortion standards

- Procurement practices and regulatory environment

- ASD acceptance by the end user (education, misconceptions, etc.)

The reason for not limiting considerations to only technical issues is that as ASD technology matures, other factors, listed above, have become dominant in determining the success of ASD applications in an increasing 
number of cases. As a result, the "barriers" to ASD penetration are becoming more complex.

The next section presents a survey of ASD technology, as well as application issues. In Section 3, the ASD market is divided into three clusters and each one is described in terms of benefits derived from ASD use, application requirements and potential for growth.

Section 4 considers all factors which influence ASD growth for each of the application groups, while Section 5 outlines a proposed action plan. The key report conclusions are presented in Section 6. 


\section{REVIEW OF ASD TECHNOLOGY}

The objective of providing adjustable speed (or torque) to a load can be reached by using either DC or AC motors. For well known reasons of required motor maintenance, sensitivity to operating environment, maximum speed, power to weight ratio and several others, DC drives are not suitable for all applications of interest. For this reason, only AC drives are considered in this section and the term Adjustable Speed Drive (ASD) will be used in this section to denote $A C$ drives.

Although industrial AC drives use almost exclusively induction motors, synchronous motors may have an advantage at very low and very high power ratings (for example, servo drives for robotics or drives above $10,000 \mathrm{hp}$ ). For that reason, AC drives discussed here include both induction and synchronous motors.

While there are several methods which can be used in adjusting the speed of an induction motor, only the method by which motor input frequency and voltage (or current) are controlled yields the required performance. Furthermore, that is the only method for continuous control of the speed or torque of industrial synchronous, motors. Therefore, only an adjustable frequency/voltage (or current) method will be considered here.

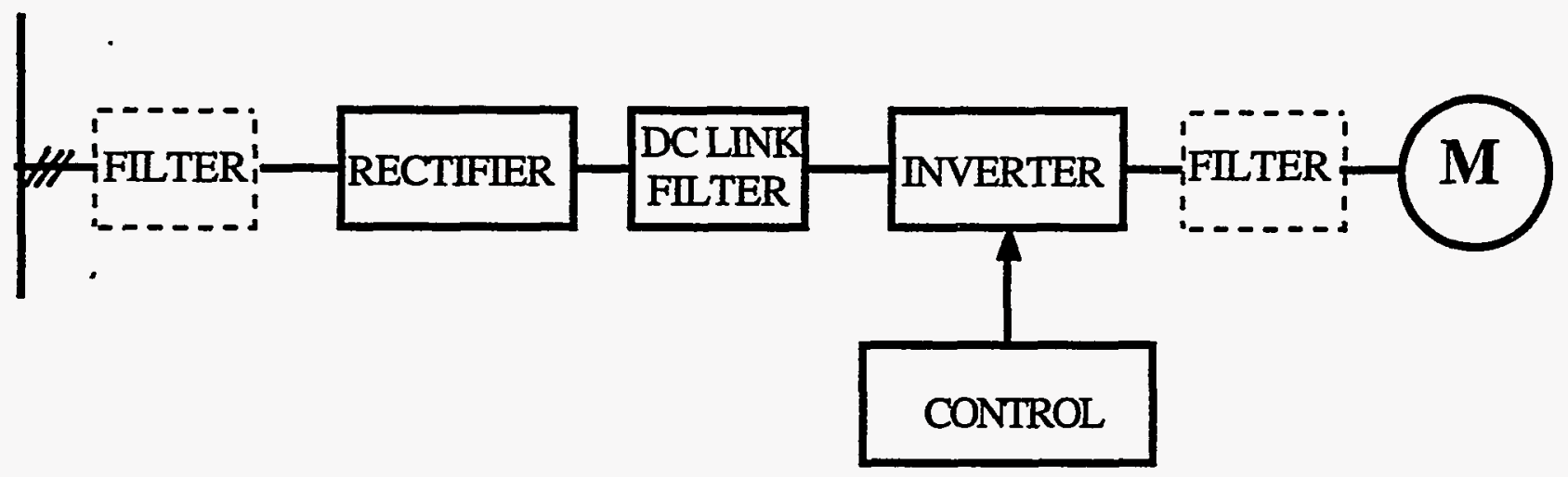

FIG. 1: GENERAL BLOCK DIAGRAM OF A TYPICAL INDUSTRIAL ADJUSTABLE SPEED DRIVE INPUT AND OUTPUT FILTERS MAY OR MAY NOT BE REQUIRED DEPENDING ON THE APPLICATION. 
With the present and foreseeable technology, the most important adjustable frequency/voltage method consists of rectifying the $60 \mathrm{~Hz}$ utility power into DC power and inverting the $\mathrm{DC}$ power back into $\mathrm{AC}$, but with adjustable frequency and voltage (or current), Fig. 1. The other method uses cycloconverters for a direct conversion of $60 \mathrm{~Hz} \mathrm{AC}$ to adjustable frequency $A C$ power. This method is found in very high power, low speed applications. It is restricted to approximately a $15-20 \mathrm{~Hz}$ output frequency when using phase commutated thyristor switches and is not economically practical with any other power switches. For that reason, cycloconverters are not covered in this section.

The family of industrial ASDs is shown in Fig. 2. Traditionally, ASDs are divided into low voltage (to $600 \mathrm{~V}$ supply) and medium voltage $(2300 \mathrm{~V}$ and above) groups. Furthermore, ASDs can be voltage source type, having a capacitor across a DC bus or current source, having a choke (inductor) in a DC bus. Each of these categories is now considered.

\subsection{LOW VOLTAGE ASDs}

These drives represent by far the majority of the total ASD population, accounting for approximately $90 \%$ of all AC drive sales in the US. This is primarily due to the number of application opportunities in the medium and low power range and because developments in power semiconductors have brought dramatically reduced ASD prices while increasing ASD performance and reliability. The technology of low voltage ASDs has consolidated on voltage source drives. A typical low voltage ASD is shown in Fig. 3.

\section{A low voltage ASD usually consists of:}

- INPUT FILTER which prevents ASD generated harmonics from entering into the utility power system. ASD power rating, type, and application as well as the "stiffness" of the electrical grid at the connection point determine whether the input filter is necessary as well as its size.

- INPUT RECTIFIER which converts $60 \mathrm{~Hz}$ AC power into DC power. If the rectifier uses diodes, the ASD operates with constant DC link voltage. If the rectifier uses thyristors, the DC link voltage can be varied.

- DC LINK which connects the rectifier with the inverter and which has a filter to smooth out the rectified voltage and to decouple inverter generated harmonics from the power grid. The size of this filter is smaller if a diode rather than a thyristor rectifier is used. 


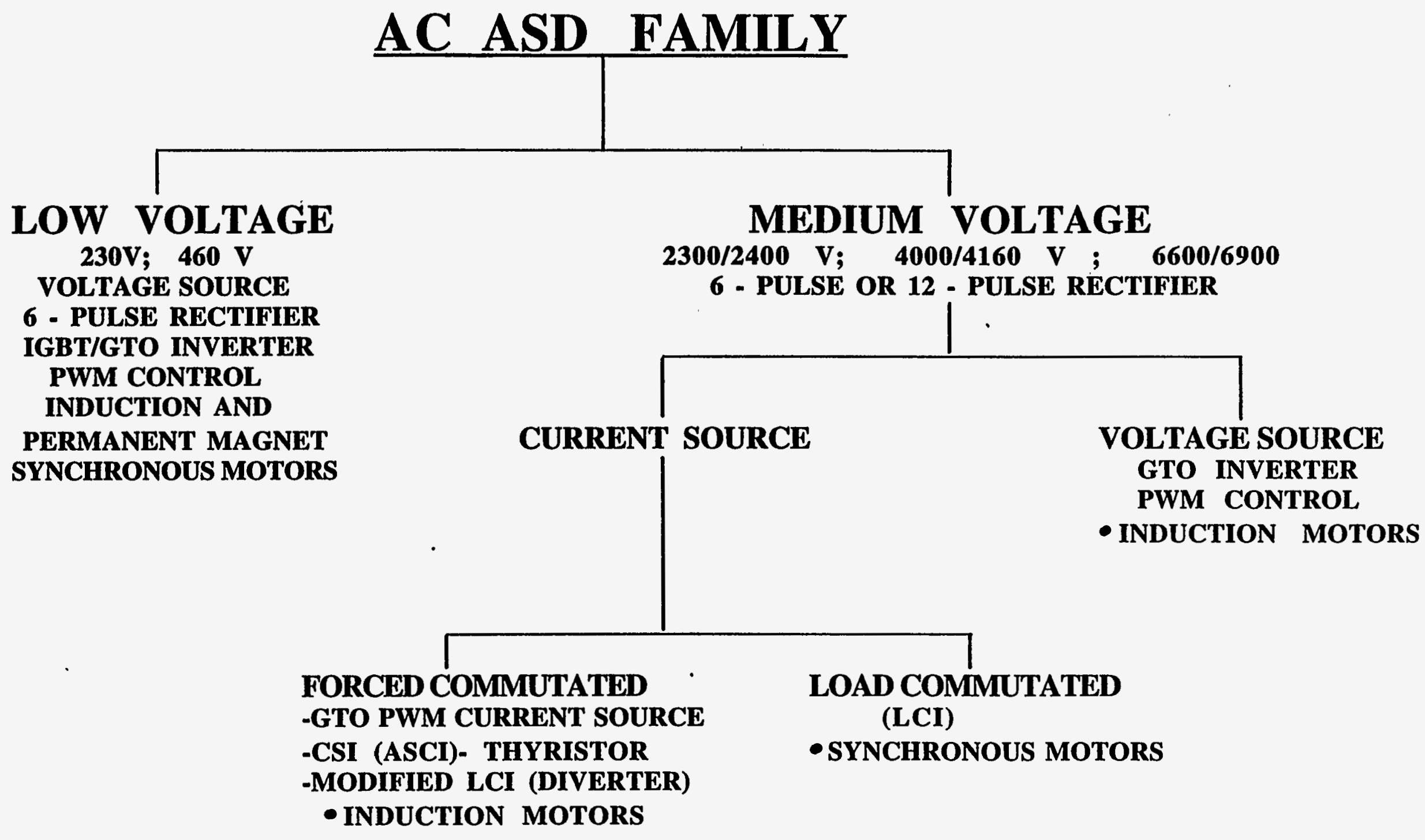

FIG. 2: INDUSTRIAL AC ADJUSTABLE SPEED DRIVES 


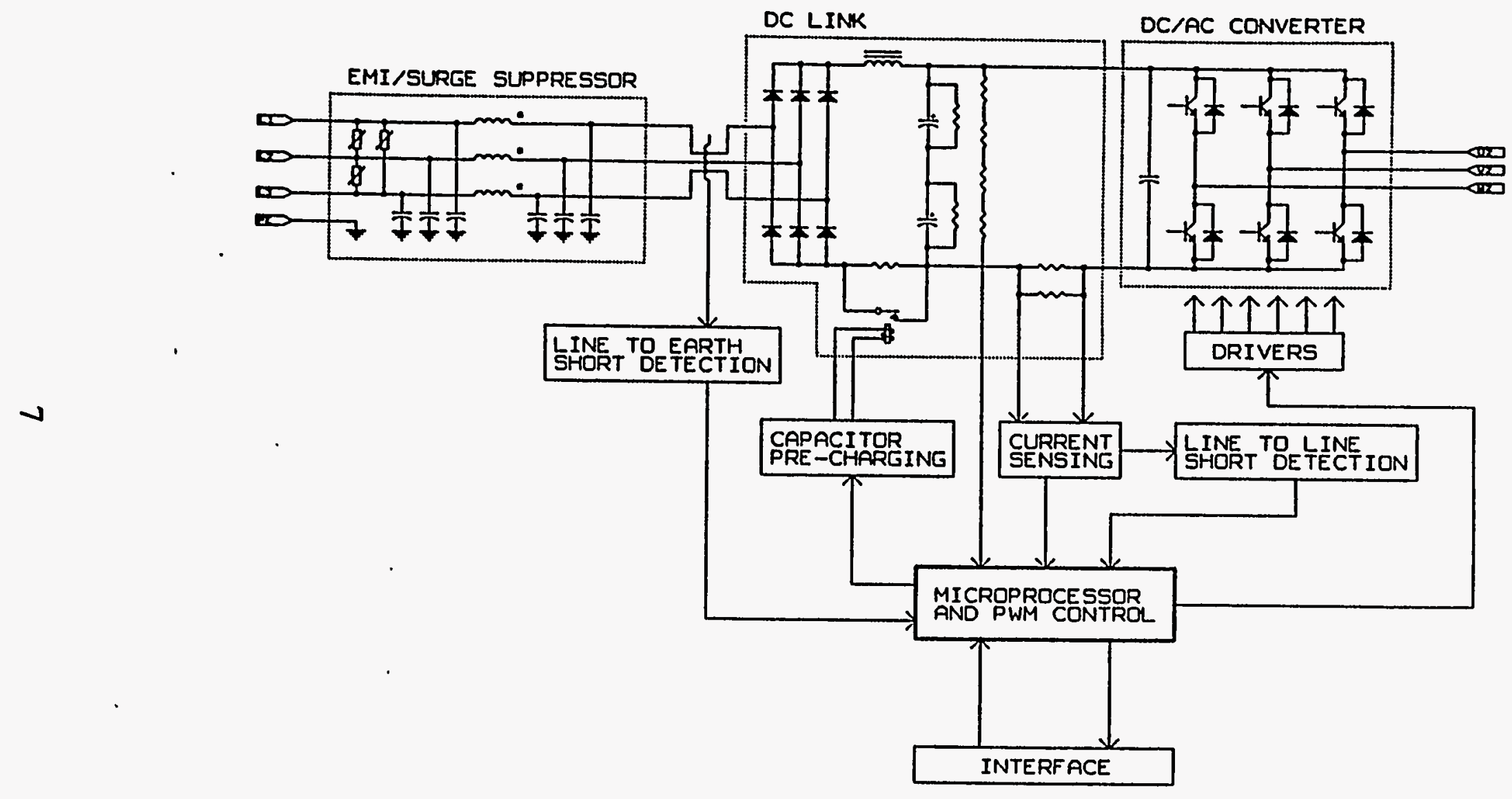

FIG. 3: CIRCUIT DIAGRAM OF A TYPICAL LOW VOLTAGE, GENERAL PURPOSE, PWM CONTROLLER ASD. 
- INVERTER, which synthesizes three-phase AC wave forms of a desired frequency from the DC link voltage. If the inverter controls both the output frequency and voltage, it is normally called a PWM (Pulse Width Modulated) inverter due to the shape of its output voltage, Fig. 4. The switching instants of the inverter semiconductors, Fig. 3, are determined by the intersection of the triangular carrier and each of the three phase reference voltage signals, Fig. 4a. With PWM control, one can use a diode input rectifier which is more economical and reliable and which requires smaller DC link and input filters than a thyristor one. Modern (IGBT) PWM inverters provide a quasi sinusoidal motor input current, with relatively small harmonic distortion. However, PWM inverters require gate-controlled power semiconductors, such as bipolar junction transistors (BJTs), insulated gate bipolar transistors (IGBTs) or gate turn-off thyristors (GTOs).

If the inverter controls only the output frequency, the voltage control has to be done by the input rectifier, which, therefore, has to use thyristors. This results in a DC-link voltage which varies according to load requirements. In this case an input filter is normally necessary, while the size of the DC link filter is increased. One calls such an inverter a "six-step" inverter (or, sometimes a VVI - variable voltage input inverter), because of the shape of the output voltage, Fig. 5. Depending on the speed range and power rating, a six-step inverter may require an output filter or motor de-rating.

Six-step inverters represent older technology which does not require fast, gate controlled semiconductor power switches (because the inverter switching frequency is much lower than for PWM inverters, Figures 4 and 5). As such, six-step inverters are rarely used in $460 \mathrm{~V}$ applications and are also disappearing from high power ASDs.

With low voltage drives standardizing on PWM control, IGBT inverters, with switching frequencies of $5-12 \mathrm{kHz}$ are used below approximately $500 \mathrm{hp}$, depending on drive over-load rating. Drives above $500 \mathrm{hp}$ use either IGBT switches in parallel, multiple IGBT inverters in parallel or GTO inverters with a switching frequency of $300-800 \mathrm{~Hz}$. (Normally, for the same motor leakage inductance, the higher the inverter switching frequency, the closer the inverter output current approximates a sine-wave). Note that the division between IGBT and GTO inverters is changing, with the share going to GTO inverters declining. 

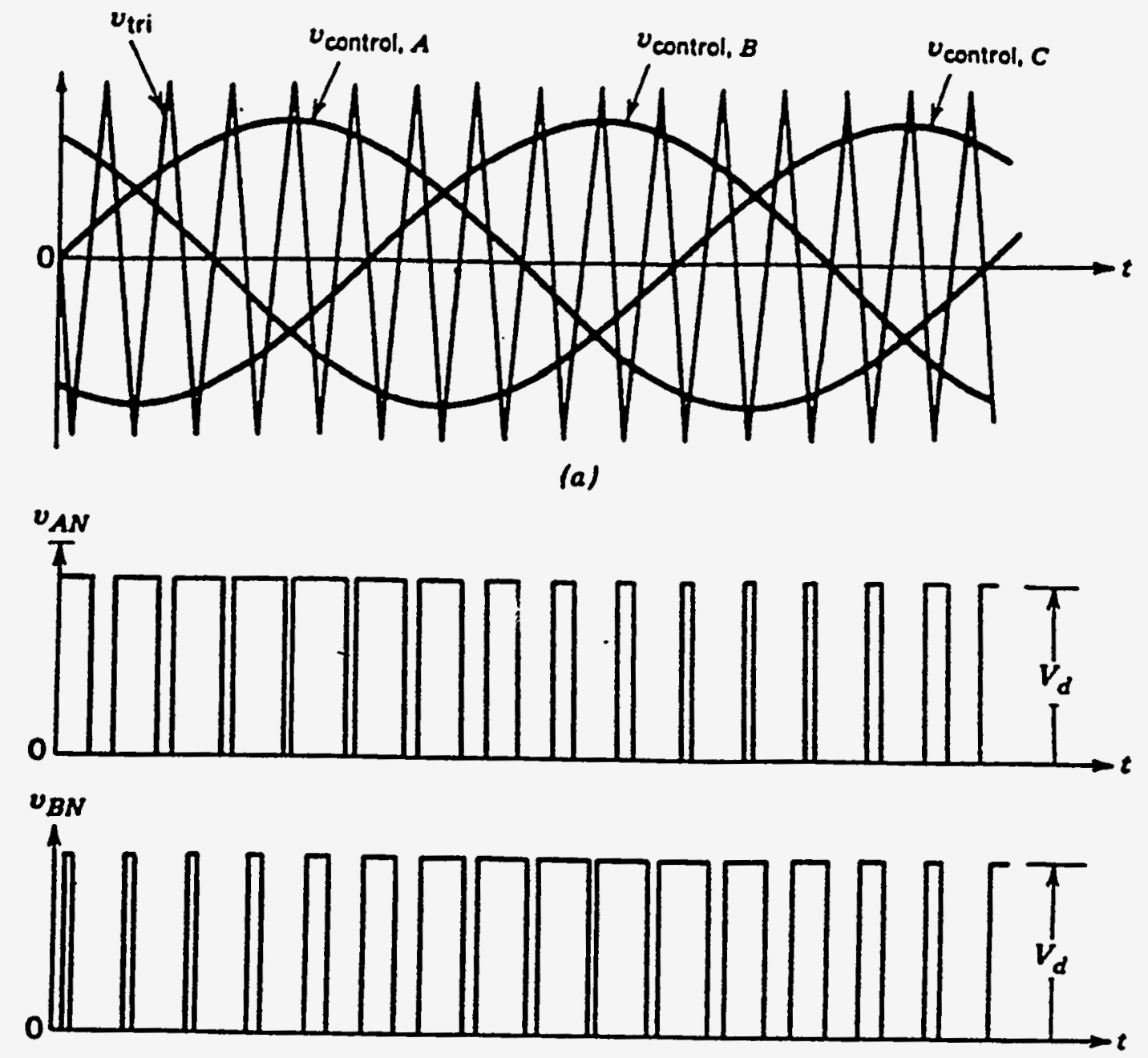

$v_{A B}=v_{A N}-v_{B N}$

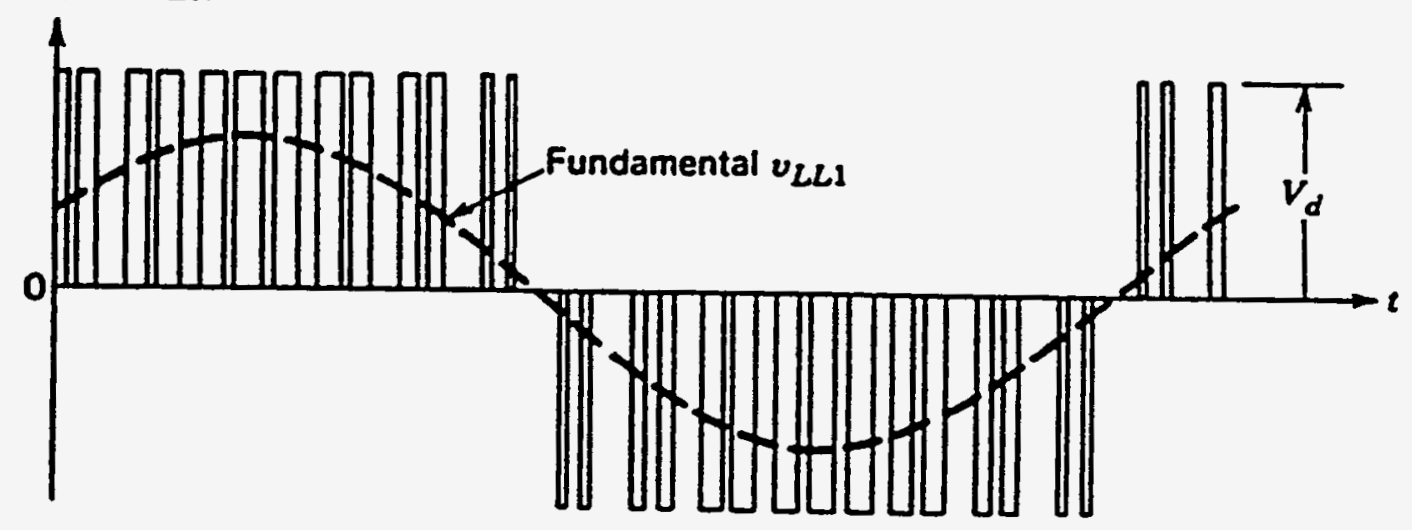

(b)

FIG. 4: THREE-PHASE PWM VOLTAGE CONTROL

(a) TRIANGULAR CARRIER WITH REFERENCE VOLTAGES

(b) LINE TO LINE OUTPUT INVERTER VOLTAGE 

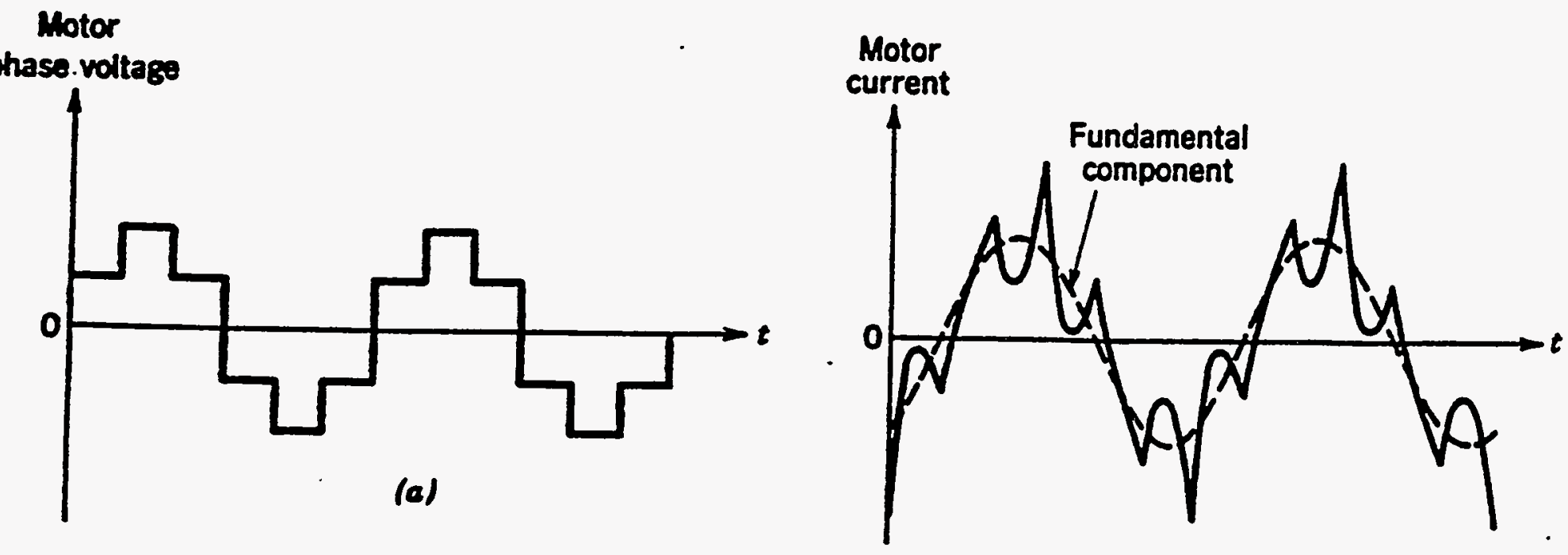

(b)

FIG. 5: SIX-STEP VOLTAGE WAVE FORMS

(a) MOTOR PHASE VOLTAGE

(b) MOTOR INPUT CURRENT 
- MOTOR which provides the load with desired speed or torque. In motion control, where position is the controlled variable, permanent magnet synchronous motors are used in closed loop, high performance servo drives. Stepper motors, usually operating in open loop are used in low torque, less dynamically demanding servo applications. In all other applications with AC drives, induction motors are used. While numerous new motor structures (switched reluctance, synchronous reluctance, brushless doubly fed, interior magnet, etc.) have appeared, none has found significant industrial use. In order to accommodate increased losses caused by harmonics in the inverter output, the motors are often de-rated, using a 1.15 service factor. This is especially true if output filters are not used.

- OUTPUT FILTER which blocks inverter generated harmonics from entering (and thus heating) the motor and which may be necessary with six-step inverters.

- CONTROL LOGIC which assures that the motor input voltage and frequency correspond to the motor speed required by the load. In addition, the control provides all protection, diagnostics and communication functions.

\subsection{MEDIUM VOLTAGE ASDS}

For motors above approximately $1000 \mathrm{hp}$, the voltage ratings become $2300 / 2400 \mathrm{~V}, 4000 / 4160 \mathrm{~V}$ and $6600 / 6900 \mathrm{~V}$, Fig. 2. At these voltage and power ratings, the use of IGBTs is no longer feasible and several ASD topologies presently exist. While they all use a rectification - DC link inversion principle, their realization yields different characteristics.

While low voltage ASDs have standardized on voltage source inverters, medium voltage drives use both voltage and current source topologies with the majority of installations being of the current source type. A brief description of both voltage and current source ASDs for medium voltage applications follows.

2.2.1 VOLTAGE SOURCE ASDs have essentially the same topology as low voltage drives, Fig. 3. (Regardless of their ratings, voltage source ASDs can always be recognized by the presence of a capacitor in a DC-link filter and free-wheeling diodes connected in anti-parallel across inverter switches). All voltage source ASDs need additional circuitry when regeneration to a utility grid is required. Because of the increased power, an input isolation transformer is normally necessary between the utility connection and the rectifier, Fig. 1. Depending on the inverter control, 
voltage source ASDs can be either PWM or six-step. Their respective characteristics are:

- PWM controlled, with a GTO inverter and a diode input rectifier. The GTO switching frequency is normally below $500 \mathrm{~Hz}$, often requiring an output filter. In order to reach the required voltage ratings, single devices (GTOs, diodes and DC-link filter capacitors) are connected in series. The resulting increase in cost limits this ASD configuration to approximately $5000 \mathrm{hp}$ maximum.

- Six-step inverters with thyristor input rectifier. Since the switching frequency and the inverter voltage stresses are lower than in PWM inverters, in principle, the power rating can be increased. However, this ASD type requires heavy input and DC link filters and often an output filter and is therefore slowly being replaced by other technologies.

One should also mention the existence of various multilevel inverter topologies $[1,2]$. In addition to full DC voltage, they use also intermediate voltage levels, available at the series connected DC link capacitors, to provide a multi-stepped inverter output voltage, Fig. 6 . In this way, for the same output harmonic distortion, the PWM frequency can be significantly lower than in conventional voltage source ASDs. One of the multilevel schemes, called Neutral Point Clamped (NPC) inverter, using three voltage levels, Fig. 6, has particular promise in high power drives [2,3]. It is schematically shown in Fig. 7. Since the medium voltage inverters need to use power switches connected in series in any case, the NPC topology does not impose additional cost penalty. Such ASD topology has been offered by $\mathrm{ABB}$ for the last four years, with its output limited to $2300 \mathrm{~V}$. This product has been targeted primarily for new installations, where a new motor can be matched with NPC ASD characteristics: like any voltage source inverter, the NPC ASDs rely on motor leakage inductance to filter out the voltage harmonics from the input current. High efficiency motors, already installed in high power applications, operate with little magnetic saturation and have low per unit leakage inductance. As such, they are ideally suited for current source ASD retrofits, but experience increased losses when voltage source (including NPC) ASDs are used. For that reason, high power voltage source ASDs essentially require either an output filter or appropriately matched motor for a successful application.

Whatever the inverter topology and control, voltage source ASDs always use induction motors in medium voltage applications, Fig. 2. 


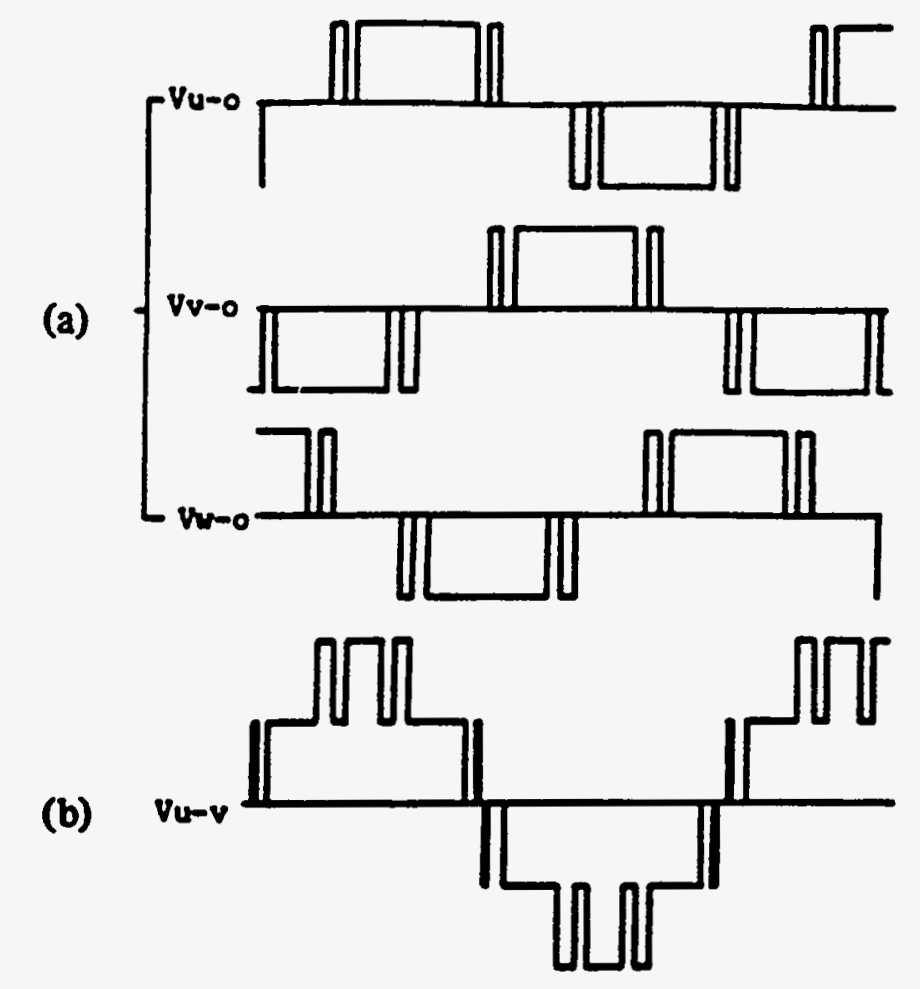

FIG. 6: EXAMPLE OF OUTPUT VOLTAGES OF A THREE-LEVEL PWM INVERTER

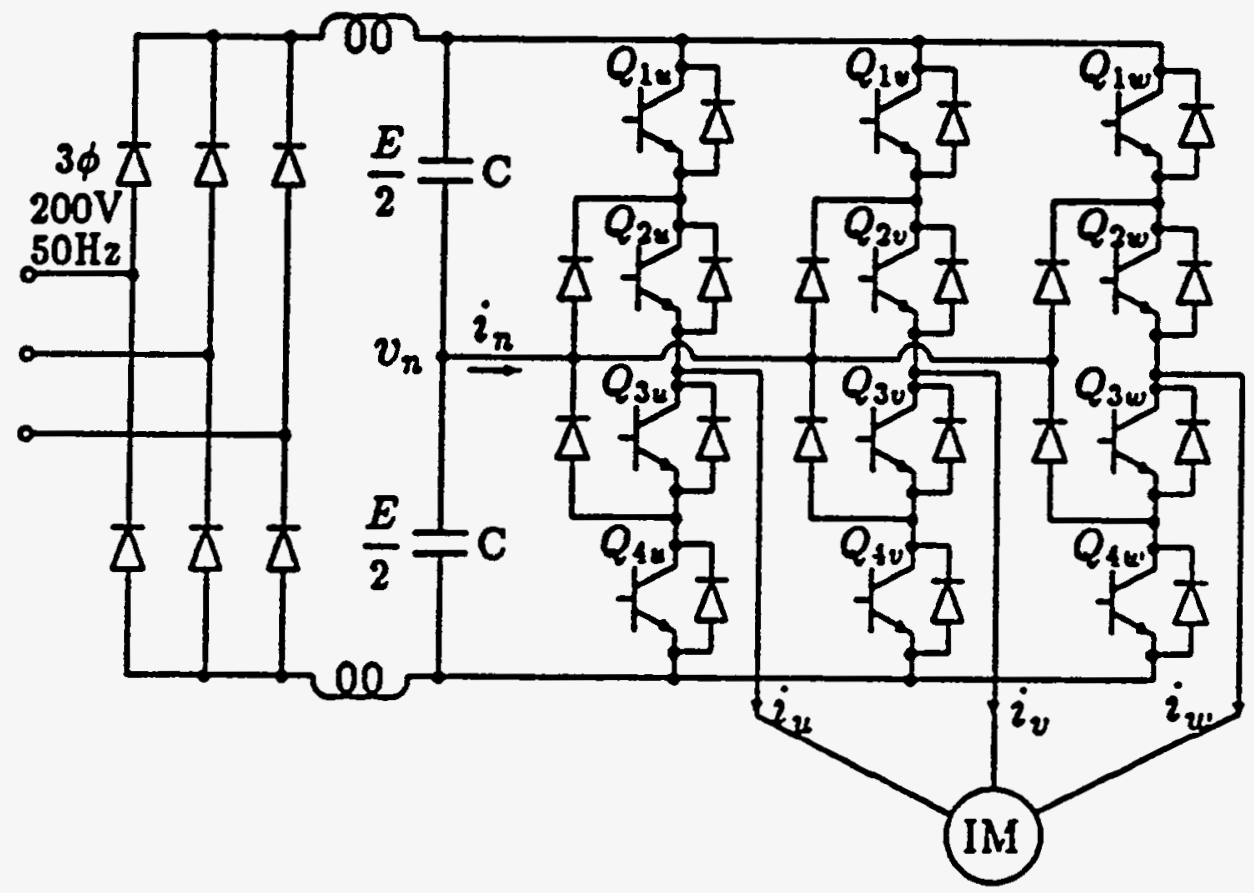

FIG. 7: CONCEPTUAL SCHEMATIC OF THREE-LEVEL, NEUTRAL POINT CLAMPED (NPC) ASD 
2.2.2 CURRENT SOURCE ASDs are recognized by a heavy inductor (choke) in a DC-link, which can reach the size of the motor itself, and an absence of a DC link capacitor and inverter free-wheeling diodes. All current source ASDs require a thyristor input rectifier which also regulates the ASD (and, thus, the motor) input current. In fact, the rectifier, together with the DC link choke, forms a current source which supplies the motor with the current required by the load, Fig. 8. As such, all current source ASDs - require heavy input filters or isolation transformers. On the other hand, current source ASDs have the inherent capability to regenerate back to the power grid during motor braking. With current source ASDs, the inverter's only function is to connect the appropriate motor phase to the current source, with a desired motor frequency determined by the switching of the inverter current steering semiconductors. Since the motor phase current is interrupted at each phase commutation, current source ASDs work best with as low a motor leakage inductance as possible, as explained above. It happens that very large, energy efficient motors, designed to operate with a low level of magnetic saturation, naturally satisfy this requirement and therefore make current source ASDs the preferred choice for retrofit applications. In fact, commutation of inverter switches represents a challenge at these power levels and several methods for their turning off have evolved. The only difference between various current source ASDs comes from the method used to commutate inverter switches. Basically two groups exist, force commutated and load commutated, which are discussed below:

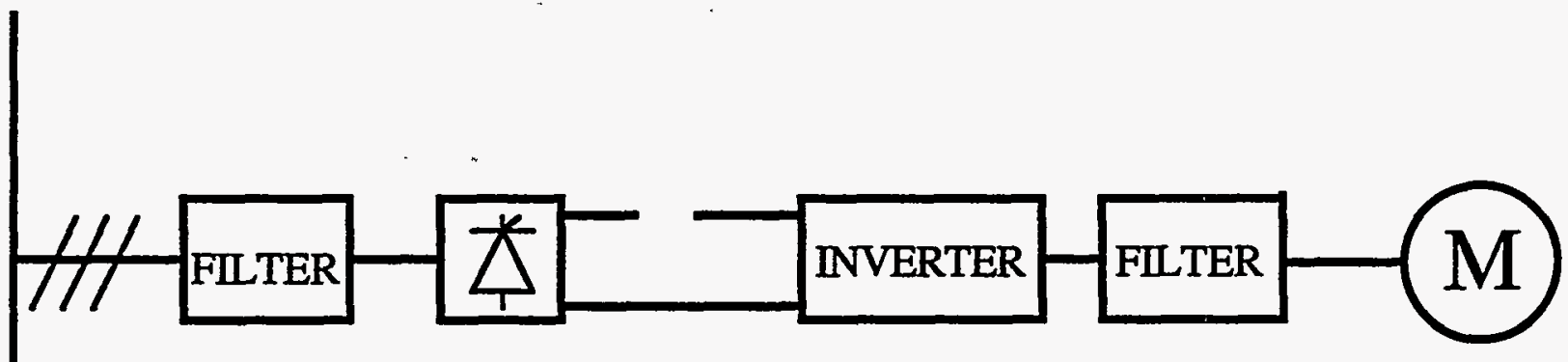

FIG. 8: BLOCK DIAGRAM OF A CURRENT SOURCE ASD. NOTE THE DC LINK CHOKE AND THE CONTROLLED INPUT RECTIFIER 
2.2.2.1 Forced commutated inverters have power switches which can be turned off irrespective of the load power factor. Basically, three types are presently used:

- Thyristor current source inverters (also called Auto-Sequentially Commutated Inverters - ASCI) use a capacitor commutation circuit to turn off the inverter thyristors and series diodes in order to keep the capacitors properly charged, Fig. 9. It is the oldest current source inverter technology [4-6], now being displaced by other concepts. Its main disadvantages are limited output frequency, limited voltage rating, cost of external commutation circuit and significant output harmonics. Its main advantage, use of thyristors for inverter operation, is losing its importance with the development of new, gate turn off switches.

- Modified load commutated inverter (Modified LCI) also uses thyristors in the output inverter, Fig. 10, and relies on two concepts [7] to turn the thyristors off:

- below approximately $30 \mathrm{~Hz}$ output frequency, the diverter circuit, Fig. 10, instantaneously shorts the DC link, diverting the current from the inverter, every time any of the inverter thyristors needs to be turned off. (The DC choke limits the rise in the short circuit current through the diverter.)

- above approximately $30 \mathrm{~Hz}$, the motor load starts to operate with a leading power factor due to the capacitors connected across motor terminals, Fig. 10. The leading power factor in turn allows for load commutation of the inverter thyristors in a range from $30 \mathrm{~Hz}$ to maximum motor speed.

Modified LCI ASDs represent the most widely used technology in medium voltage $(2300-6600 \mathrm{~V})$ pump, fan, and compressor applications. This technology is presently the cost leader in 2000 $10,000 \mathrm{hp}$ ASDs although the situation may change as better GTOs and new power semiconductors are developed.

- GTO PWM current source ASDs, Fig. 11, represent the most advanced force commutated current source technology in the sense that it is the most silicon intensive concept with no additional circuits to turn off the inverter switches. The capacitors connected across the motor terminals are much smaller than those in Fig. 10 and serve to absorb the energy stored in the motor leakage inductance during each commutation, [8-10] 


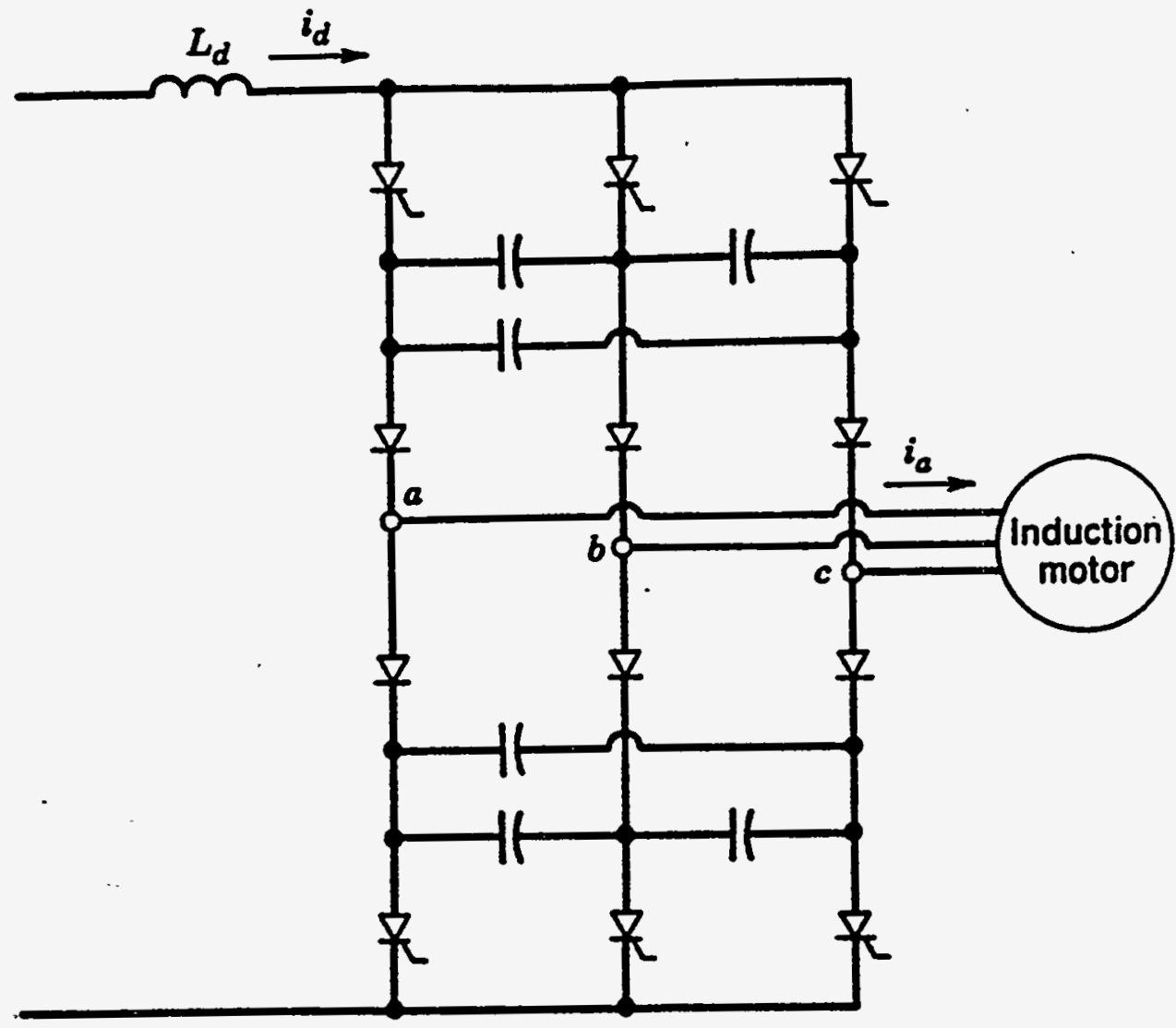

FIG. 9: AUTO SEQUENTIALLY COMMUTATED CURRENT SOURCE INVERTER (ASCI) 


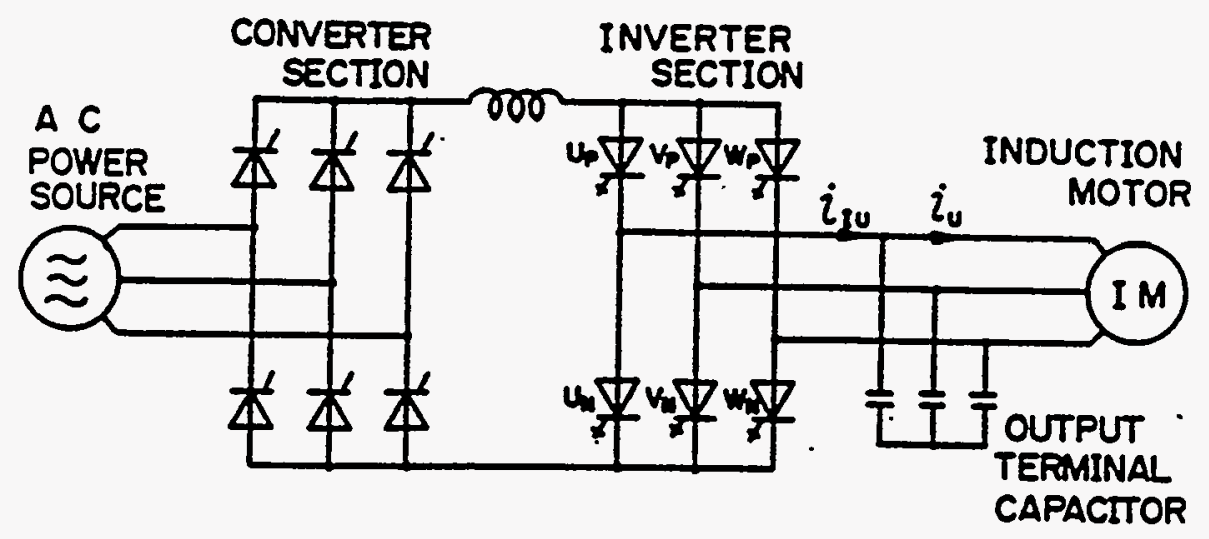

Main circuit configuration.

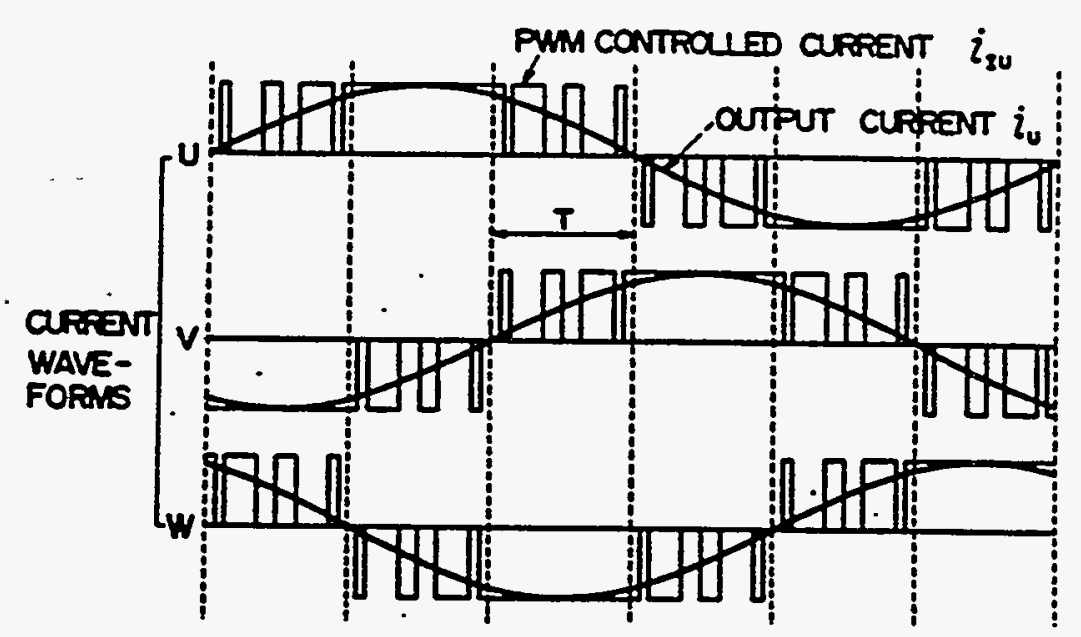

Basic operation waveforms.

FIG. 11: CURRENT SOURCE INVERTER WITH GTO PWM OUTPUT CONTROL 
This type of ASD provides the best (least distorted) wave forms to the motor, as the PWM control is used to reduce the output harmonics.. With further improvements in GTOs, this technology is expected to eventually become a price leader, starting at the low end of the medium voltage range.

All current source, forced commutated ASDs use induction motors.

2.2.2.2 Load commutated inverters (LCIs), Fig. 12, use standard thyristors in the inverter bridge and depend on the motor leading power factor for thyristor commutation. For that reason, LCI ASDs can be used only with over-excited synchronous motors. In this case, the ASD operates in the same way as a high voltage DC (HVDC) transmission line - on the rectifier- side, it is connected to a power system (utility grid) of a given (60 $\mathrm{Hz}$ ) frequency; on the inverter side, it is tied to another $\mathrm{AC}$ power system (synchronous machine) of different (variable) frequency. Therefore, the power range of an LCI ASD is determined primarily by economics rather than by technical limitations and LCI ASDs are found in applications with ratings of 5,000 - 40,000 hp, [11]. (Below approximately 8,000-10,000 hp forced commutated ASDs normally become more economical.)

In order to maintain synchronism between the inverter and the motor during both steady state and transient conditions, inverter switching is made dependent on rotor position, usually through a rotor position feedback, Fig. 12. In fact, the inverter can be viewed as a solid-state commutator, performing the same function as the mechanical commutator on a DC machine. The position sensor obviously increases the ASD cost while decreasing its reliability.

\subsection{ASD CONTROL}

Drive control determines ASD performance with respect to torque per ampere, transient response, and steady state accuracy. With the availability of powerful microprocessors and the development of advanced control techniques, control and the resulting drive performance are no longer limiting factors in ASD applications. In order to optimize the motor output (i.e. torque/amp ratio) it is desirable to operate the motor as close as possible to its rated flux, while the motor input frequency and the shaft load vary. Each control method tries to reach this objective - the differences come from control complexity/sophistication, the information required to implement it and the end result. While there are many variations, ASD control can be basically divided into these two groups: 


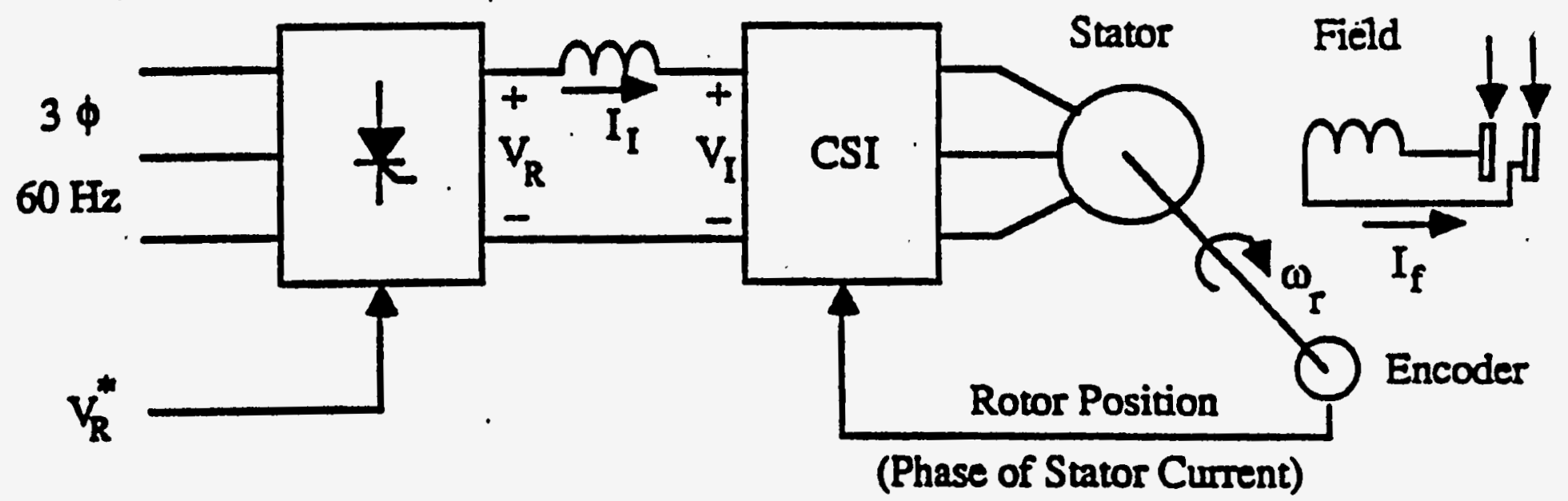

FIG. 12: LOAD COMMUTATED INVERTER. NOTE ROTOR POSITION FEEDBACK WHICH ENSURES SYNCHRONISM AT ALL LOAD CONDITIONS 


\subsubsection{CONSTANT VOLTS/Hz CONTROL}

With this control, the motor voltage is made proportional to the motor frequency, Fig. 13. (Some voltage biasing is normally provided at low speed to improve motor torque capability.) In this way the motor magnetizing current (and thus the motor flux) can be maintained approximately constant in steady state and at a rated load. Load compensation, based on current measurement in a DC link, may provide a range of constant flux operation around the rated load. However, since control implementation is constrained by cost considerations, the compensation is usually never completely effective and, since no motor variable is directly measured, the flux varies significantly from the rated value during speed and load transients. As a result, the drive dynamic performance is poor. Nevertheless, the constant $\mathrm{V} / \mathrm{Hz}$ control is the only one used providing generally acceptable operation in all pump, fan and compressor (PFC) applications, which are very price sensitive. However, it is expected that the new generation of sensorless ASDs, with some form of field oriented control, may replace constant $\mathrm{V} / \mathrm{Hz}$ drives in most $\mathrm{PFC}$ applications, in order to improve motor efficiency and utilization, [12].

\subsubsection{FIELD ORIENTED (VECTOR) CONTROL}

This type of control allows independent regulation of motor flux and torque, thus providing an induction motor with the same control characteristics of a separately excited DC motor. This is highly desirable as it provides an optimal motor utilization by controlling the motor to operate at its rated flux level. Furthermore, having a direct control of the motor torque, the outer speed loop (closed outside of vector control) yields very good dynamic response. To achieve this objective, vector control requires the following:

- Instantaneous control of the motor three phase input currents, rather than the motor voltage. This means that a current regulator with motor current feedbacks is required. Current control is implemented in low voltage ASDs by using voltage source inverters with PWM control to produce the desired current. In medium voltage ASDs with current source inverters, current control is accomplished in the front-end rectifier with the help of a DC link choke.

- Knowledge of motor electrical parameters. Depending on the particular vector control implementation, one may need the value of each parameter of the motor equivalent circuit or only stator resistance, inductance and magnetizing current. 
(V)

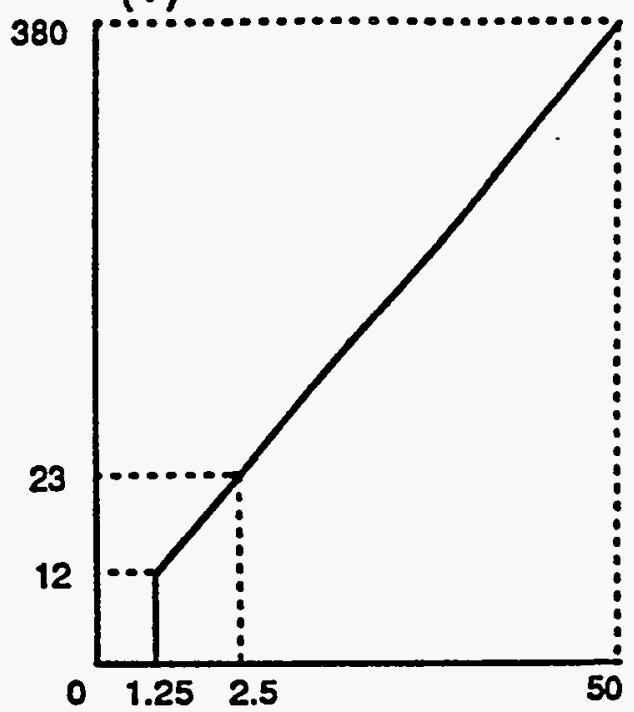

(V)

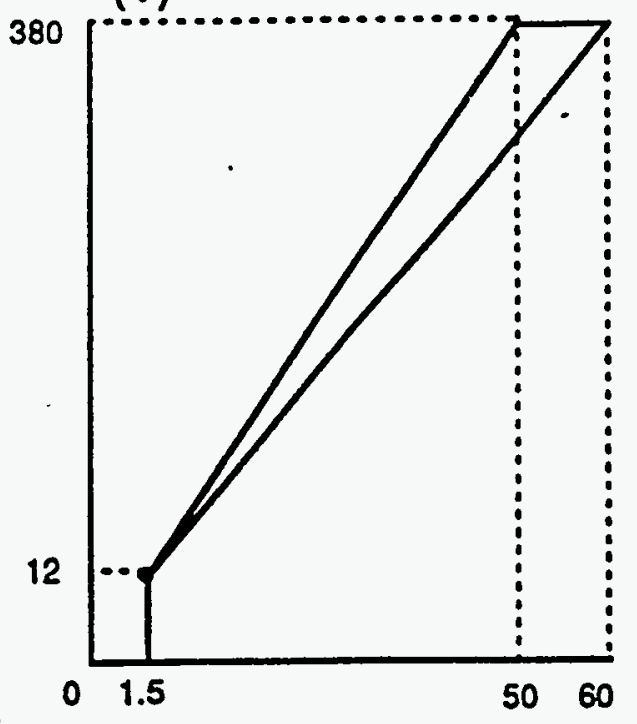

$(\mathrm{Hz})$
(V)

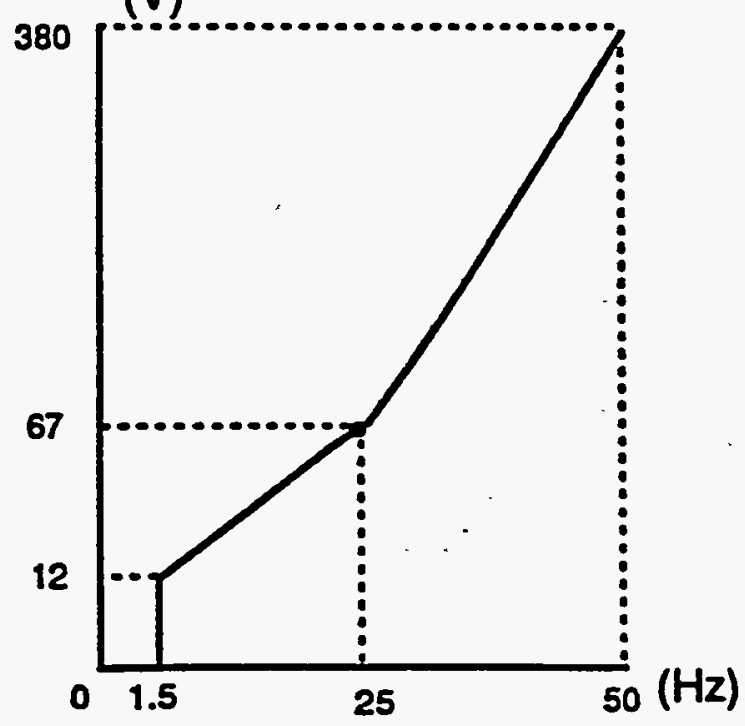

(V)

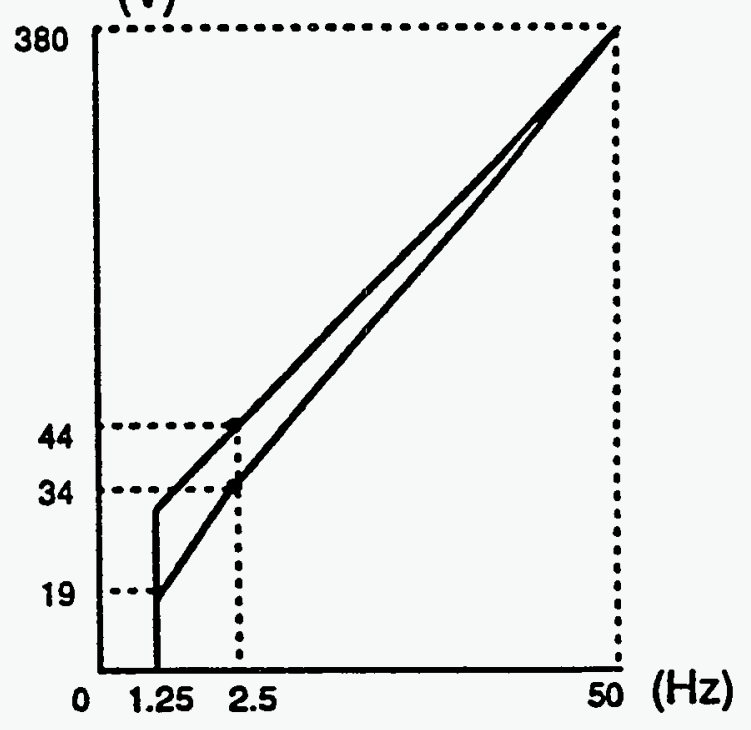

FIG. 13: SEVERAL EXAMPLES OF CONSTANT V/HZ CURVES. USER SELECTS V/HZ RELATIONSHIP IN FUNCTION OF APPLICATION 
- Correct implementation of a fairly complex control algorithm. Such implementation is essentially not feasible without a microprocessor.

There are two basic types of vector control [13,14]: direct and indirect. Direct field oriented control, Fig. 14, integrates the motor terminal voltage to obtain information about the motor flux. As such, Direct Control works well to $1-3 \mathrm{~Hz}$ output frequency but cannot provide torque control at standstill. The Indirect Control, Fig. 15, imposes the required motor slip which yields independent control of motor flux and torque. The Indirect Control works very well at standstill and is regularly used in servo applications but requires information about rotor position, normally obtained through a shaft mounted encoder or resolver. Correct operation of Indirect Control strongly depends on accurate knowledge of rotor resistance and inductance, both of which vary during motor operation.

Presently, the distinction between Direct and Indirect Control is becoming less important since, implemented in an ASD microprocessor, they complement each other over the speed range [15], when zero-speed torque control is required. Otherwise, only Direct Control is normally used.

Derivatives of Direct Control, [16], requiring a limited knowledge of motor stator parameters (obtained through ASD self-commissioning tests, [17]) are slowly replacing constant $\mathrm{V} / \mathrm{Hz}$ control in all but the most price demanding applications.

Finally, some of the new control techniques, such as sliding mode, fuzzy logic, neural networks, etc., might add marginal improvements to performance already available with field oriented control. The main benefits of these techniques would be to make vector control less sensitive to changes in motor parameters and, separately, to allow for a smooth transition between a current regulated mode and a six-step operation. Such a transition extends the maximum speed range of vector controlled drives in the motor field-weakening region. 


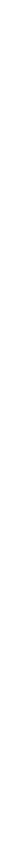

$$
\begin{aligned}
& \lambda_{r}=\lambda_{d r}=L_{m} i_{d s}+L_{r} i_{d r} \\
& \lambda_{q r}=0 \\
& T=\frac{3}{2} \frac{P}{2} \frac{L_{m}}{L_{r}} \lambda_{d r} i_{g s} \\
& \left(R_{r}+L_{r} p\right) i_{d r}=-L_{m} p i_{d s}
\end{aligned}
$$$$
\begin{aligned}
& V_{d o}=R_{s} I_{d s}-\omega L_{s}^{\prime} I_{q s} \\
& V_{q o}=R_{s} I_{q s}+\omega L_{s} I_{d s} \\
& L_{s}^{\prime}=L_{s}-\frac{L_{m}^{2}}{L_{r}}=L_{\delta s}+L_{\delta r}
\end{aligned}
$$

FIG. 14: DIRECT FIELD ORIENTED CONTROL WITH SPEED AND FLUX CONTROL LOOPS AND CURRENT REGULATORS IN SYNCHRONOUS REFERENCE FRAME 


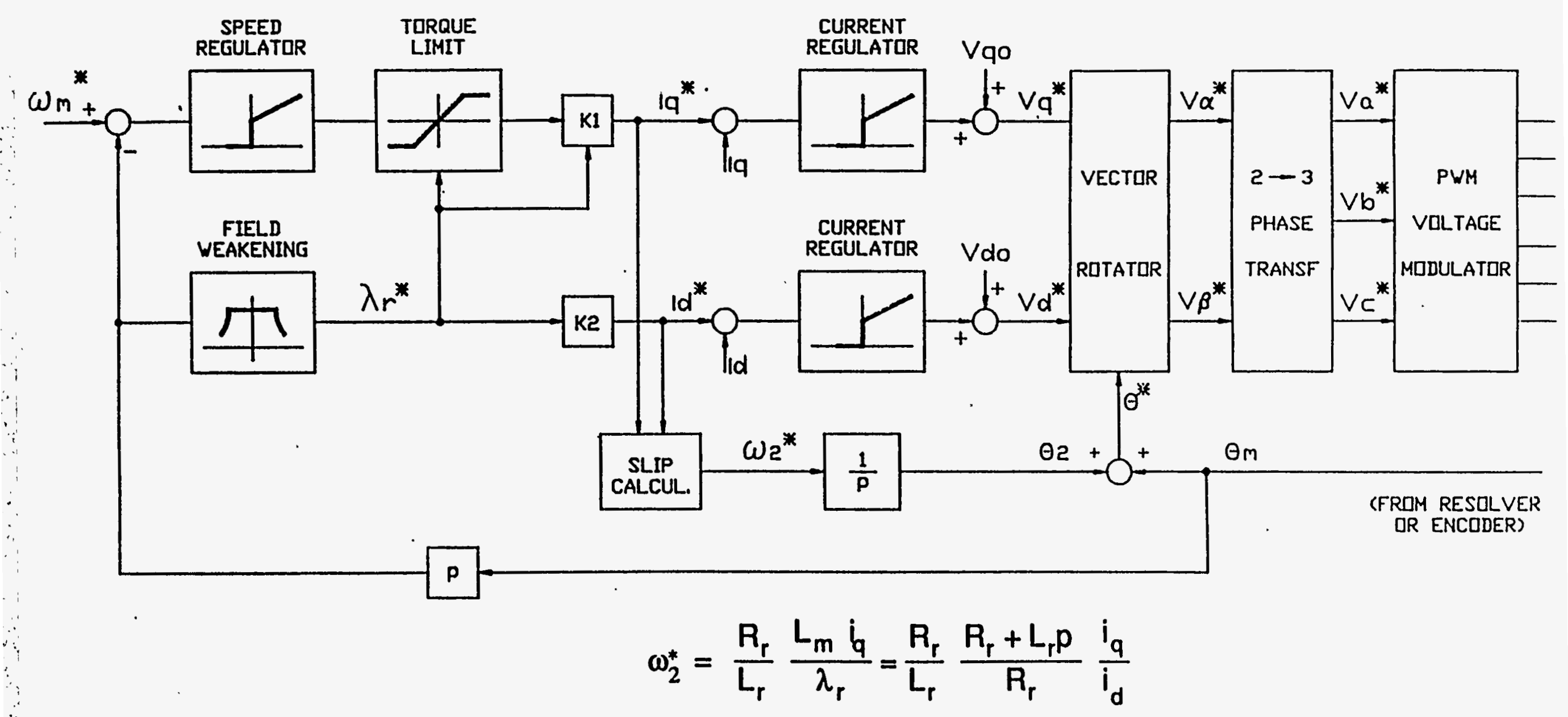

FIG. 15: INDIRECT FIELD ORIENTED CONTROL WITH CURRENT REGULATORS IN SYNCHRONOUS REFERENCE FRAME 


\subsection{ASD TECHNICAL ISSUES}

The use of electronics 'and the switching operation of static power converters generate problems with ASD applications which are not present when a motor operates connected directly to the power grid. These problems can be broadly grouped into these four categories:

- Harmonics injected into power grid.

- ASD sensitivity to power quality problems.

- ASD output harmonics.

- Motor insulation stress.

Each of these categories is now discussed briefly.

\subsubsection{HARMONICS INJECTED INTO POWER GRID}

Every front-end rectifier in present day industrial ASDs draws nonsinusoidal currents from the electric power grid. These currents may cause line voltage distortions, depending on the grid output impedance at the point of ASD connection and on the ASD power rating. Current distortion (current harmonics) cause additional losses in the power grid; the resulting voltage distortion may cause faulty operation of other equipment, supplied by the distorted voltage as well as resonance problems in the power grid. Although current distortion is more pronounced with phase-controlled thyristor rectifiers operating with large firing angles, diode rectifiers also result in input current distortion. The following measures may be used to reduce input current harmonics:

- Use of DC choke with DC link filter capacitor in voltage source ASDs, Fig. 16. The choke smoothes down the "peaky" input current and also helps to filter the much higher frequency harmonics produced by inverter switching.

This approach is used primarily in low voltage, PWM controlled ASDs.

- Use of filters connected between the input rectifier and the power grid, Fig. 1. Such filters are used to trap specific harmonics (each filter is tuned to one harmonic). With thyristor rectifiers supplying voltage source ASDs, the filters become complex and bulky. With medium voltage current source ASDs, the input filters are often replaced by an input isolation transformer.

- Use of two, series connected rectifiers supplied from two separate, delta and star connected transformer secondaries, Fig. 17. In this way, the 5th. and 7th. (the most significant) harmonics are 


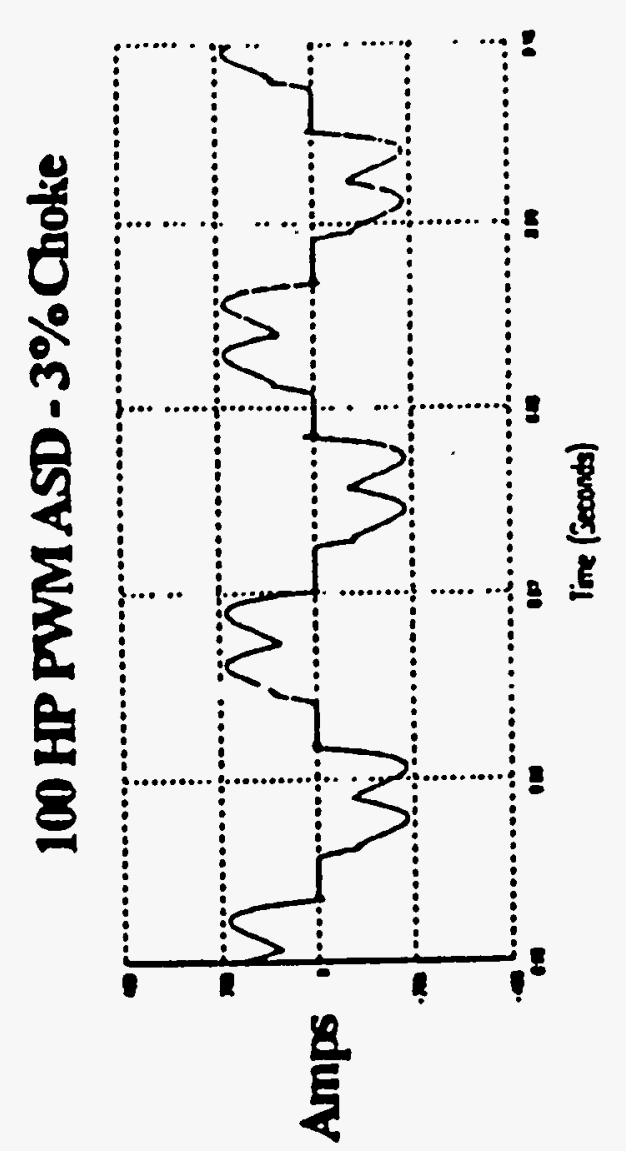

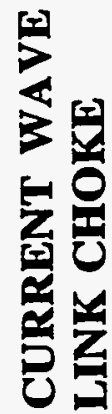

을

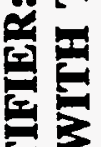

불

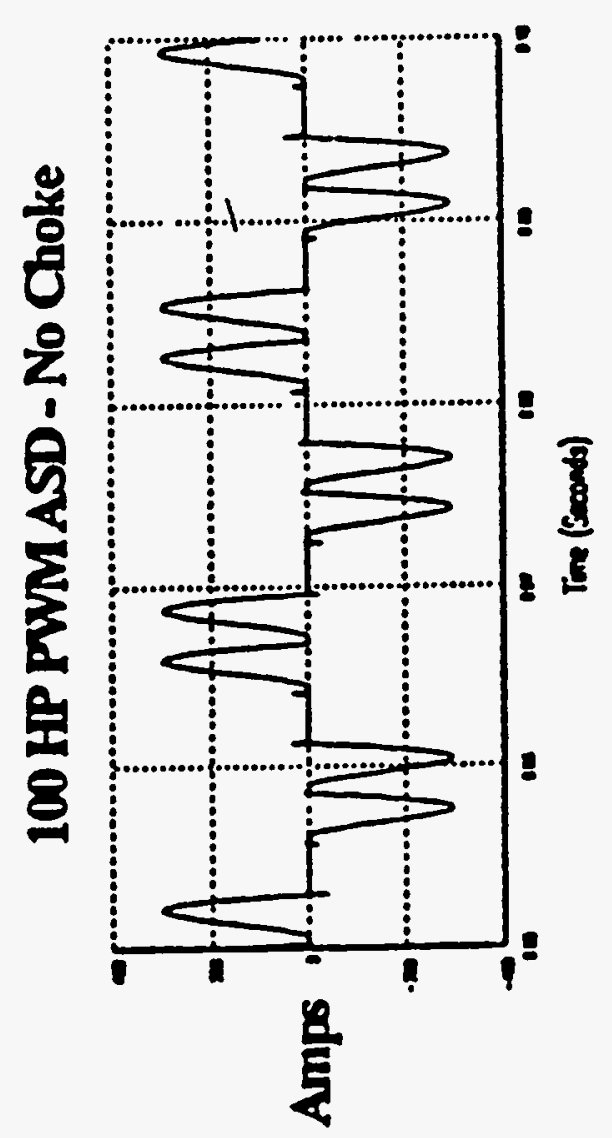

봉응

$\ddot{\theta}$
$\ddot{0}$ 


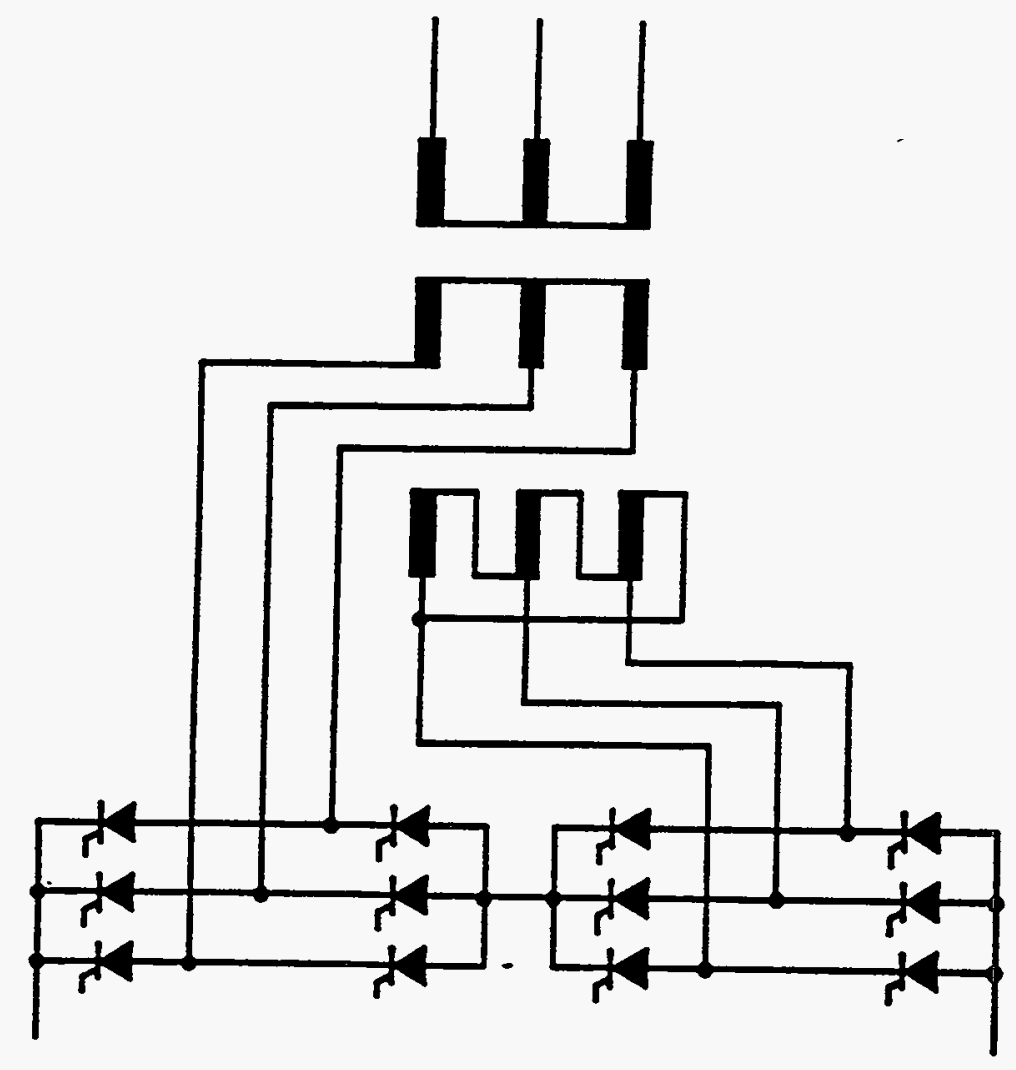

FIG. 17: 12-PULSE CONFIGURATION OF AN ASD INPUT RECTIFIER. BY USING DELTA-STAR TRANSFORMER SECONDARIES, THE INPUT VOLTAGES TO THE TWO RECTIFIERS ARE PHASE SHIFTED BY $30^{\circ}$. AS A RESULT, ALL 5th AND 7th HARMONICS ARE CANCELED FROM THE AC INPUT WAVEFORMS, ASSUMING BALANCED CONDITIONS. 


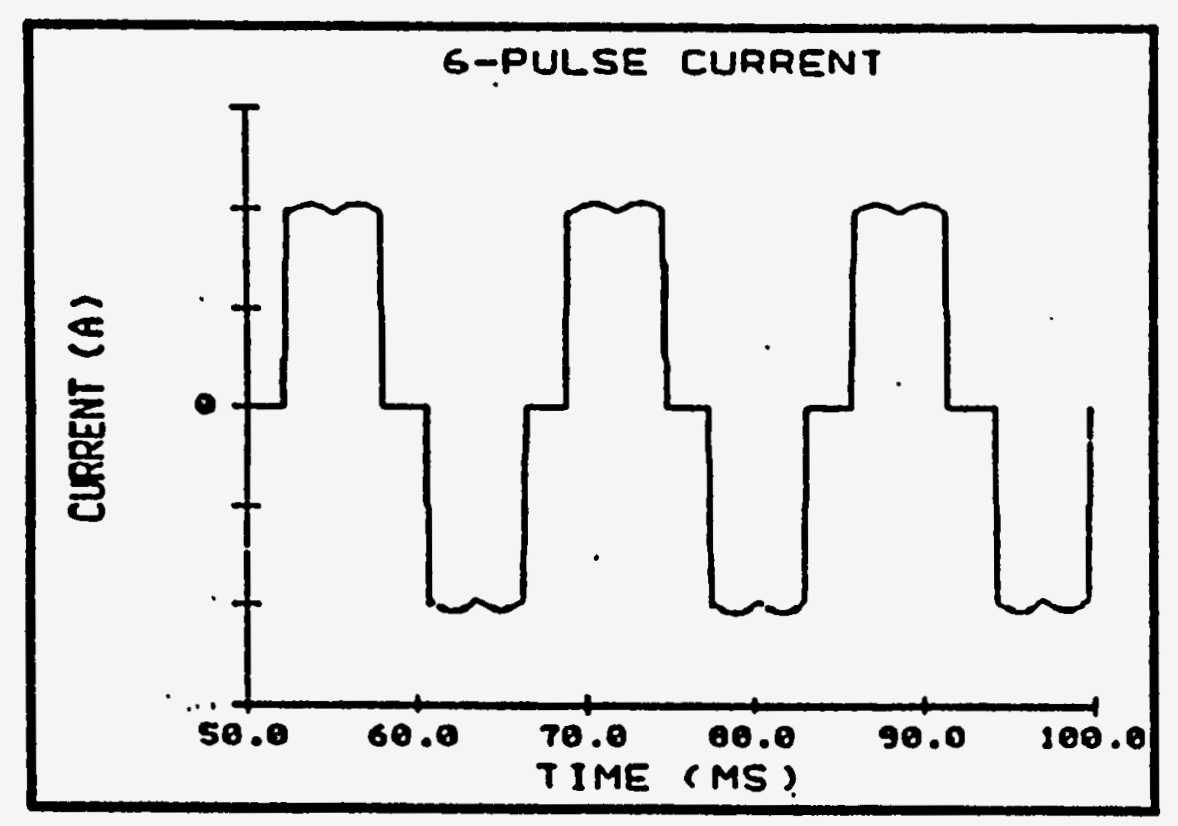

6 pulse: $h=6 n \pm 1$

$(5,7,11,13, \ldots$.

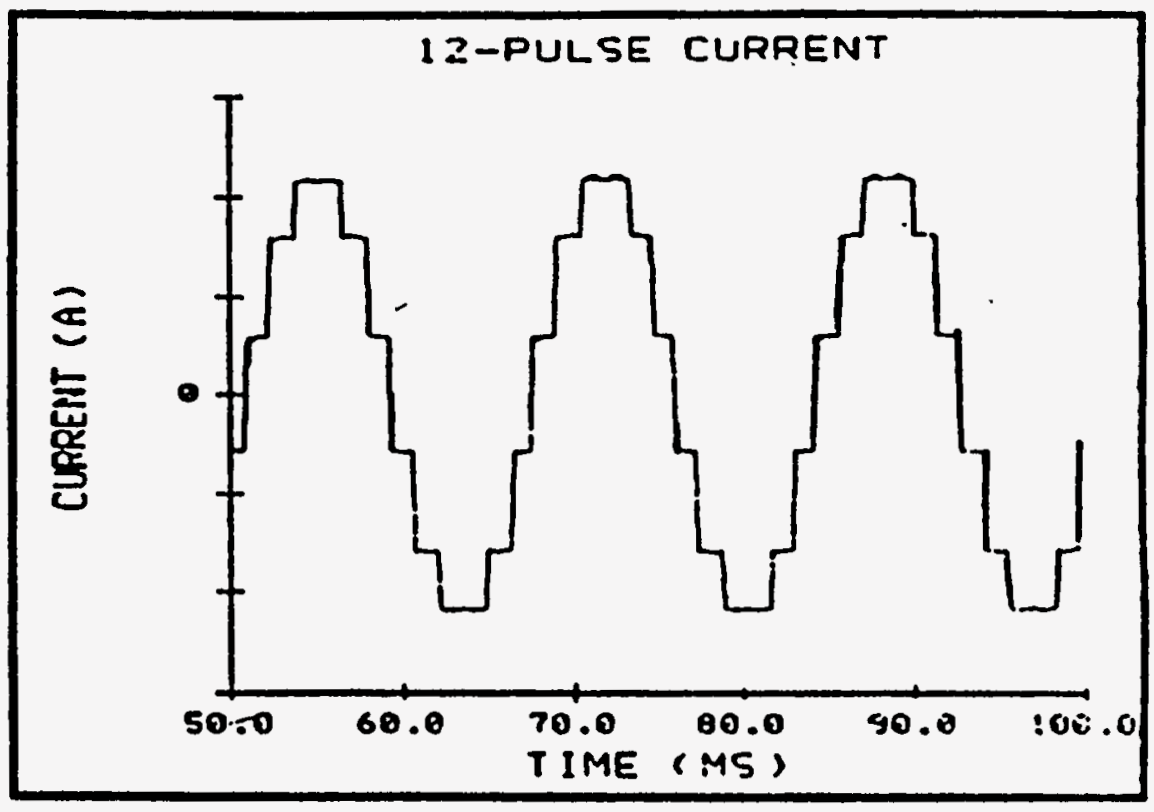

12 pulse: $h=12 n \pm 1$

$(11,13,23,25, \ldots .$.

FIG.18: ASD INPUT CURRENT

(a) WITH 6-PULSE RECTIFIER

(b) WITH 12-PULSE RECTIFIER, SUPPLIED FROM

DELTA-STAR TRANSFORMER SECONDARY 
eliminated and the input current is made to more closely approximate sine-wave, Fig. 18. This method is obviously suitable only for high power ASDs.

All methods to eliminate or reduce harmonics in ASD input currents add cost and require additional space. Yet, as the number and the cumulative installed power of ASDs continue to grow, the harmonic distortion problem also grows, becoming less tolerable. An approximate rule is that the harmonic distortion becomes an issue when the total non-linear loads (such as static power converters) reaches $25-40 \%$ of the total facility load. This situation is being addressed by new regulations (such as IEEE 519) which limit the harmonic distortion which can be injected into utility grids. While regulations which limit harmonic distortion are not yet legislated, their application is often requested by the end user. Compliance with these present and future regulations increases the ASD cost and may represent, in the future, one of the main impediments to ASD growth.

\subsubsection{ASD SENSITIVITY TO POWER QUALITY PROBLEMS}

While ASDs affect power systems through harmonic distortion of their input currents, power systems impact ASDs in ways that are not common when the motor is connected directly to the power grid. As a result, ASDs may trip-out and fail in situations in which a motor, súpplied from a utility grid, would have no problems operating normally. The following two power quality problems are the most common affecting ASDs:

- Instantaneous voltage dips, usually caused by faults elsewhere in a power system. During a fault, the voltage at the ASD connection point may drop to $20-80 \%$ of its rated value, depending on the fault type, its distance and grid configuration. Since it takes a circuit breaker 3-5 cycles $(50-83 \mathrm{msec})$ to clear a fault, ASDs may see a voltage dip lasting 100 - 300 msec. Because a typical, non-hardened ASD will trip at $80 \%$ of the rated voltage after $15-50 \mathrm{msec}$, ASDs will experience frequent trippings during power system faults. These trippings may be extremely harmful to a particular ASD load and have recently caused great concern. (Note that a motor connected to utility lines will normally ride through these voltage sags due to the combined motor load mechanical inertia, as long as the contactor remains latched). Since power line faults are not controllable, the following measures are normally taken to secure ASD ride-through capability in critical applications: 
- Operating ASD control and logic from a UPS (Uninterruptible Power Supply) while adjusting the under-voltage tripping levels.

- Braking with regeneration to DC bus in applications with large inertia loads. In this way, the load kinetic energy is returned to the DC bus, supporting the bus voltage during utility voltage sags. With this scheme, the power supply for logic and control is obtained from the DC bus.

- Using additional DC link capacitor banks to support the DC link voltage during voltage sags. This approach has a very limited effectiveness.

- Voltage surges, caused by a switching of power factor capacitor banks, load shedding, lightning, etc. The voltage surges may cause failure of the input rectifier or ASD over-voltage trippings in case of surges having limited peaks but long duration. The most effective protection from voltage surges is the use of $A C$ reactors, DC link chokes or oversizing of rectifier components.

\subsubsection{ASD OUTPUT HARMONICS}

ASD output is not sinusoidal and harmonics injected into a motor cause additional motor heating (core and additional copper losses due to skineffect ) as well as torque pulsations. The extent of the harmonic distortion in the ASD output current depends strongly on the inverter type used. For example, IGBT inverters with PWM control, switching over $4-10 \mathrm{kHz}$ can normally use a standard induction motor without any filtering or motor derating, basically taking advantage of the motor's 1.15 service factor. GTO PWM voltage source inverters switching at $300-800 \mathrm{~Hz}$ would normally use a larger frame motor, effectively derating the motor. Less frequently in low voltage ASDs, an output filter may be used. At medium voltage, a low inverter switching frequency as well as the importance of motor efficiency normally require use of some type of output filter. The situation is somewhat helped by the fact that these large motors have a high winding $L / R$ ratio which contributes to harmonic filtering.

\subsubsection{MOTOR INSULATION STRESS}

The insulation dielectric stress may be caused by excessively high, repetitive terminal voltage and/or by the extremely fast rise times of the PWM voltage wave forms (excessive $\mathrm{dv} / \mathrm{dt}$ ). The motor insulation failure mechanism is only now starting to be understood after repeated field experiences with "cold," dielectric failure of motor insulation. The two problems are mutually independent, although they are both caused by fast 
switching of inverter devices (such as IGBTs) and both typically result in insulation stress of the first turn of the first coil of the motor winding.

The first problem, excessive motor voltage, occurs due to a resonance in the cables which connect the motor to the ASD, [18]. The resonance occurs when each voltage switching (PWM pulse) takes longer than half the pulse rise time to travel from inverter to motor. In that case, the amplitude of the voltage pulse at the motor terminals will have approximately double the rated value. Of that, about $80 \%$ of the peak amplitude will develop over the first turn of the first coil. That means that the motor is subject to this voltage stress at $4-10 \mathrm{kHz}$ continuously during its operation, for the normal life of the motor.

The problem obviously occurs for combinations of cable lengths and inverter switching speeds. It has been found, [18], that over-voltage problems can occur for cable lengths up to $100 \mathrm{~m}$ and rise times of up to 2 usec. The remedies for this problem are either slowing down the rise time of the PWM pulses (through snubbers across inverter devices, output filters or IGBT gate control), limiting the cable length or reinforcing the motor insulation. The first approach is contrary to the modern trend of faster PWM switching; the second is limited by the physical layout of a particular application, and the third negates the idea of using an "off the shelf, standard induction motor."

The second problem is even more subtle than the first and can be ascribed to leakage currents through the insulation to ground.

In each motor winding there are small, distributed, parasitic capacitances between winding turns and between the winding and the motor frame. These capacitances play no role until the rise time of the applied voltage becomes sufficiently short to cause capacitor currents to flow. This happens with most IGBT and some BJT inverters when the rise time of the PWM pulses starts to exceed $5-6 \mathrm{kV} / \mathrm{us}$. (The exact value depends on the type of motor winding, that is, on parasitic capacitance.) Fig. 19 shows a measured leakage current to ground for an ASD switching at $5 \mathrm{kHz}$, [19] The leakage currents would first trip differential (ground fault) relays, making ASD installation and commissioning very difficult in the field. If the problem is not corrected (by slowing down inverter switching or by use of a common mode output filter, [19]) the leakage currents cause accelerated insulation aging and eventually insulation puncture-through. Field reports indicate that in some cases the failure can occur after only 1 2 weeks of ASD operation. These reports also indicate that some motors may operate for months while motors from other manufacturers fail 


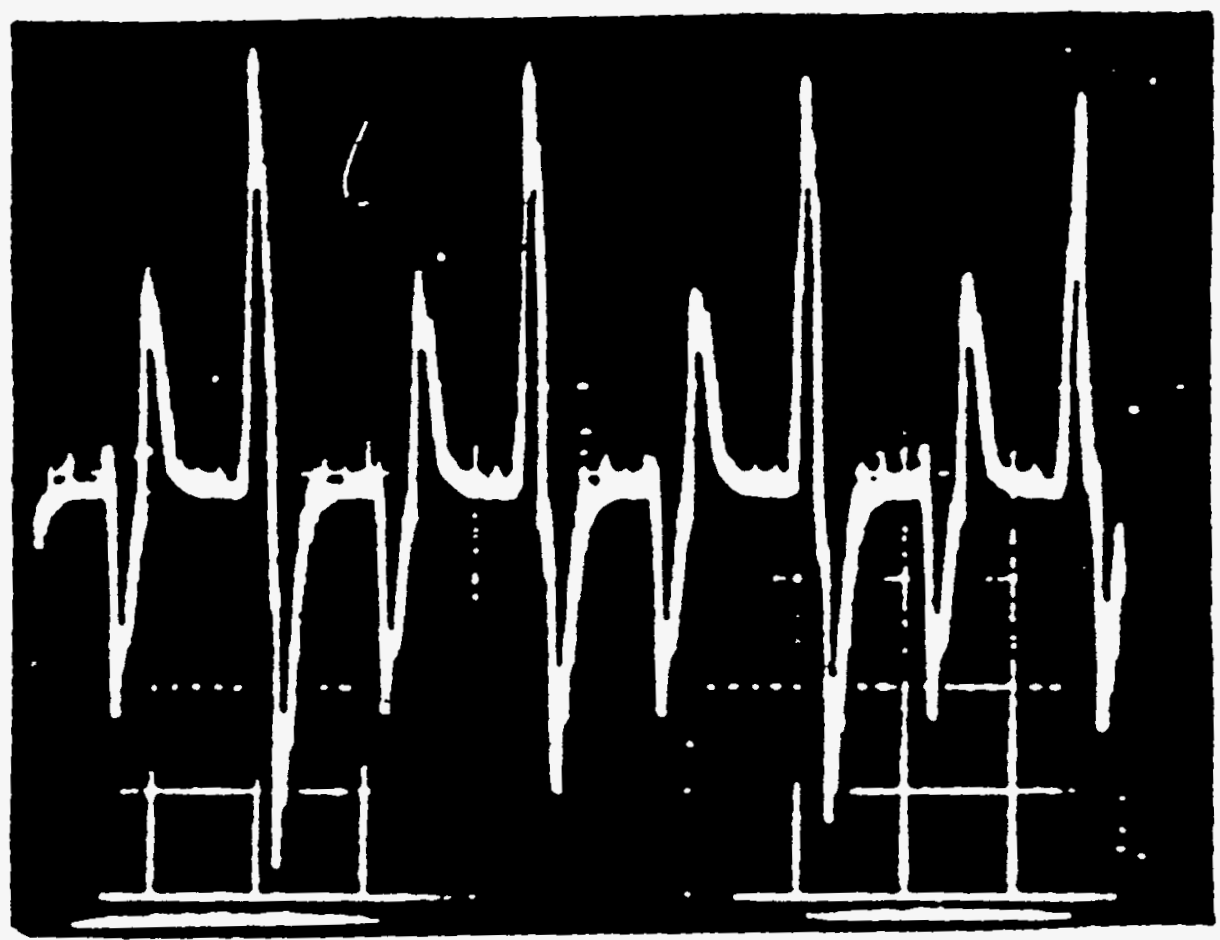

$20 \mu \mathrm{sec} / \mathrm{DIV}, 10 \mathrm{~mA} / \mathrm{DIV}$

FIG.19: INDUCTION MOTOR LEAKAGE CURRENT TO GROUND, HAVING THE SAME FREQUENCY (5 KHZ) AS THE CARRIER, AND CAUSED BY HIGH DVIDT IN ASD OUTPUT PWM VOLTAGE 
within weeks, when supplied from the same ASDs. The difference may come from the quality of varnish on the magnetic wire.

The most economical remedy for the problem of motor leakage currents seems to be double (and possibly triple) varnish insulation of the magnetic wire.

\subsection{TECHNICAL SUMMARY}

- ASD technology is driven by advancements in the semiconductor industry in both the power device and signal electronics (microprocessor) areas. Future technical improvements in ASDs closely depend on future progress in semiconductor technology. This is particularly true in the case of power switches for medium voltage ASDs.

- ASD technology for low voltage (up to $460 \mathrm{~V}$ ) applications has standardized on a diode rectifier with a PWM inverter. IGBT devices have replaced or will shortly replace all other devices in applications below approximately $500-600 \mathrm{hp}$. (Depending on ASD required overload capability, single devices are used approximately to $300 \mathrm{hp}$ and paralleled IGBTs or complete inverter modules above that.)

- GTO devices are used at the high power end of the low voltage range $(600-1000 \mathrm{hp})$ and are also moving to medium voltage applications. Industrial drives are now benefiting from developments of GTO devices driven primarily by electric train applications in Japan and Europe. However, due to their low switching frequency, very low turn off gain and sensitivity to voltage transients, GTOs are far from having all desirable characteristics.

- At medium voltage applications $(2300-6800 \mathrm{~V})$, several technologies, all based on a current source concept, presently exist. PWM GTO current source ASDs (pioneered by Hitachi) and thyristor inverter ASDs with a diverter in the DC link (pioneered by Ross Hill-Ansaldo) are currently the most viable technologies. While diverter - ASD is presently the cost leader, it is expected that the future belongs to the GTO ASD.

- At very high power (above approximately $8000-10,000 \mathrm{hp}$ ) the only viable concept is a Load Commutated Inverter (LCI) using a 
synchronous motor with a leading power factor, as GTOs and forced commutated thyristors become un-economical.

- Any analysis of ASD technology must recognize the technological discontinuity between low voltage ASDs which use fast IGBTs and high power, medium voltage ASDs struggling with commutation problems due to a lack of sufficiently powerful semiconductor switches. In fact, most ASD growth over the last fifteen years has occurred in low voltage applications, driven first by bipolar transistors and then by IGBTs. Low voltage ASD technology is now maturing as witnessed by industry standardization on a single, PWM ASD concept. In medium voltage applications there is a competition among several technologies, none of which comes close to satisfying all the application requirements. Although current source PWM ASDs and diverter ASDs are relatively new concepts that have been developed over the past fifteen years, their power range and performance, respectively, fall short of application requirements. For that reason, it is important to keep in mind the differences in technical maturity between low and medium voltage ASDs.

- Control of ASDs falls into two categories, each tailored to one family of applications. Constant $\mathrm{V} / \mathrm{Hz}$ control has found wide use in pump, fan and compressor applications where dynamic response and speed accuracy are not important. Field oriented (Vector) Control is used in all high performance speed and torque regulated drives such as in the steel, paper, rubber, glass, etc. industries. Eventually, a simplified version of vector control will also be used in pump, fan and compressor applications in order to improve motor utilization and efficiency.

- Technical issues related to ASD application concern either ASD generated harmonics into the power system or motor, motor insulation stresses or ASD sensitivity to power system transients.

Of these, the problems of harmonics injected into the power system is a serious one and has the potential of limiting ASD growth in the future.

ASD sensitivity to power quality is also a widespread problem which fortunately can be resolved in a relatively economic manner. In spite of this, many users perceive the ASD sensitivity as a barrier to ASD applications. 


\section{KEY ASD APPLICATION OPPORTUNITIES}

The objective of this section is to explore present and potential ASD applications and to identify required ASD characteristics.

There are many ways in which one can classify drive applications. For the purpose of this study, division into the following three groups seems to be the most appropriate:

- Applications where speed control is required and is part of the process

- Applications where the flow rate has to be controlled, but such control is currently not performed by regulating the motor speed. Thus, speed control is not required by the process, but, if implemented, could yield benefits (such as energy savings)

- Emerging, large volume, applications which presently do not use ASDs but could be converted to adjustable speed in the future.

Such division permits the identification of ASD characteristics required by each application group. Division by various industries (as, for example, pulp and paper, petrochemical, etc.), on the other hand, lumps together general purpose ASDs with high performance ASDs, low voltage with medium voltage drives, making characterization of each group less clear. Considering, for example, the pulp and paper industry in greater detail, a typical mill will have low performance, general purpose drives in pump applications and very high performance, torque controlled drives on the finishing line and winders.

Each of these application groups is now analyzed.

\subsection{APPLICATIONS WITH MANDATORY SPEED CONTROL}

This group consists of applications where process technology requires adjustable, controllable speed. Examples are motion control (machine tool, robotics, etc.) drives, hot and cold metal rolling, all winder and coiler drives, finishing line paper mill drives, etc. This very diverse group is also one in .which ASDs have been traditionally used, with significant accumulated operating experience. However, since a large percentage of applications in this group have been already converted from hydraulic and mechanical control to electrically controlled speed, future ASD growth here is essentially tied to the growth of the respective industries in this group. The only difference would come from conversion of the few remaining processes (such as plastic injection molding, where hydraulic drives are 
still used, eddy current couplings or clutch brake and limit switch operations) to electrical speed control, providing that the ASD price is low enough. The market segment using eddy current couplings, clutches, brakes, limit switches, mechanical sheaves, etc. for speed control is estimated at $\$ 25 \mathrm{million} /$ year, for new equipment [20]. To that, one should add the retrofits to obtain the maximum up-side potential for ASD growth above the growth of underlying industries.

Within this group, however, there will be a continuous re-alignment of ASD technologies, with AC drives picking up an increasingly larger application segment, at the expense of DC drives. The average annual projected growths through 1995 are $9.5 \%$ for $\mathrm{AC}$ and $2 \%$ for $\mathrm{DC}$ drives. [21]

The key benefits derived from ASD use in this application group are process and/or product improvements. Other benefits, such as reduced energy use, decreased maintenance, longer equipment life, etc., may or may not be obtained but are secondary to the key objectives of process/product improvements. An exception to this is the case of lossy methods for speed control, which result in substantial heat generation (such as eddy current couplings) and where the process requires a controlled environment. In that case, conversion to ASDs is often justified by the reduction in generated heat and, thus, reduced air conditioning loads. Some examples follow.

\subsubsection{PROCESS IMPROVEMENTS}

\section{- Productivity Improvements}

- By increasing the process speed

Examples: - High speed machining

- Hot steel rolling

- Paper mill finishing line

- By improving the working duty cycle; e.g. by shortening the non-productive time in repetitive operations

Examples: - Punching presses

- Transfer line (engine block manufacturing)

- Pick and place robots

- By shortening or eliminating set-up times between production batches or product changes

Examples: - Car body welding and assembly

- Paper and textile mills

- Machining centers 
As a result, in addition to direct productivity improvements, process cost structure is changing, permitting the running of much smaller batches economically, as well as giving greater flexibility in batch sizes.

- Reliability Improvements

Through improved up-time and reduced maintenance cost:

- By replacing hydraulic with electric controls

Examples: - Machine tools

- Robots

- Plastic injection molding

- By integrating ASDs into overall process control

Examples: - Process status report (broken tool, out of tolerance cutting force)

- Self-tune and self check-up features

- On-line diagnostics

- Short mean repair time (MRT) through modular construction, fault diagnostics and self-tune

\subsubsection{PRODUCT IMPROVEMENTS}

\section{- Improved Product Uniformity}

- By reducing product tolerances through improved torque/speed control.

Examples: - Metal cutting (machine tools)

- Hot and cold metal rolling

- All process industries (rubber glass, plastic, etc.)

- By compensating for variations in process parameters or input raw material.

Examples: - Food industry (raw material variations)

- Petrochemical industry (temperature, viscosity, pressure, etc. variations)

- Paper industry (both process and product material variations)

Requirements imposed on ASDs by this application group are consistent with the objectives of ASD use: the process requires continuous speed or torque regulation and any deviation from the desired value usually represents a loss of process control. Generally, the quality of the drive is measured in terms of steady state precision and dynamic response. 
Specifically, characteristics and requirements of this group are:

- Drives are made for specific tasks (spindles, winders, servos, etc.). The load characteristics are usually well defined and known beforehand, permitting drive optimization for an application.

- The electronics and motor are matched by the ASD manufacturer to optimize the drive for the best dynamic performance, which typically requires a speed loop bandwidth of $5-50 \mathrm{~Hz}$. Good dynamic performance in turn requires an inner current (torque) loop.

- Field oriented (or Vector) control is becoming a norm for this application group, essentially replacing DC drives.

- Digital communications is used for fault diagnostics, drive status updates and change in process set points or even work cycles. Hardware is standardized mostly to serial link twisted pair or coaxial cable RS-232 (for distances to $40 \mathrm{ft}$. and speeds of 9.6-19.2 kbaud) and RS-485 (for distances to $1800 \mathrm{ft}$. and speeds to $200 \mathrm{kbaud}$ ). Software, however, is not standardized, except in Europe, where Profibus is being used for general purpose drives together with Interbus-S, while fiber-optic Sercos is being introduced for high performance machine tool drives.

- Motors often require a shaft extension on the non-driving end, to accommodate rotor position or speed sensors.

- Applications require full motor torque at reduced (sometimes zero) speed (constant torque load) leading to motors with independent cooling fans.

- In some applications (such as machine tool spindles), the motor is required to run with a weakened field at $300-500 \%$ of its rated speed.

- Except for occasional high power metal rolling applications, the vast majority of ASDs in this group operate off $460 \mathrm{~V}$, with most common power ratings not exceeding 300-500 hp.

- Permanent magnet synchronous (Brushless) motors are the standard in servo drives, while induction and separately excited DC motors are used in all other applications. 
- Required reliability is typically $30,000-50,000$ hours MTBF for a single ASD installation and 60,000-80,000 hours MTBF when a large number of drives is used in the same process (for example, a car body welding line with, typically, over 20 robots, each one having six servo drives).

- ASD specifications and purchasing decisions are normally made by OEM engineers who usually have good technical knowledge about drive characteristics. End-users have higher technical competence than in other application groups.

- In spite of strong price pressures, performance, reliability and interface flexibility still command a premium, as they ultimately lead to reduced system life cost. Also, OEMs, driven by end-users striving to improve productivity and make a better product, tend to look for a higher performance drive in order not to compromise the overall system performance.

In summary, applications with mandatory speed control usually require high performance ASDs, designed specifically for a given application. Future ASD growth in this group is essentially tied to the respective industry growth, since most applications have been already converted to electrical speed control. The main trend in this group is towards the use of AC drives, away from DC motors. The barriers to ASD growth in this group are analyzed in Section 4.

\subsection{APPLICATIONS WHERE SPEED CONTROL IS OPTIONAL}

This group consists of applications where various benefits can be obtained by controlling the motor speed. However, the process can function, without these benefits, with constant speed. For that reason ASDs in this application group use AC motors [11], (induction to 4000-6000 hp and synchronous, above $3000-7000 \mathrm{hp}$ ) either because ASD application is 'a retrofit to a constant speed operation, because the possibility of direct operation from a power grid is desirable as a back-up in case of ASD failure, or because it is the only technology suitable for high power, high speed applications.

Both low and medium voltage ASDs are found in this application group, with .ratings from 5-10 hp to $30,000 \mathrm{hp}$, when load commutated synchronous motor ASDs are used (described in subsection 2.2.2.2).

By far, the most important class in this application group is the one having energy savings and/or reducing load demand charges from the utility as the main objectives. Other ASD applications are, for example, acceleration of fixed speed large inertia (such as $60 \mathrm{~Hz}$ operated large synchronous 
motors, gas turbines, etc.), drives for large conveyor belts, large cranes and passenger elevators (where smooth acceleration and deceleration are important), mixers and blenders (where adjustable speed compensates for material consistency and variation in feed rates), etc.

Opportunities for above-average growth in ASD use in this group are significantly better, especially in the energy saving applications, than in the previous group, where speed control is mandatory and where ASDs have traditionally been used. The reason is that in energy saving applications, ASD market saturation is still relatively low since only a small percentage of all drives use adjustable speed.

In order to examine this further, characteristics of energy saving applications and related ASD requirements are now considered.

Energy saving is realized by using motor speed to control rates of fluid flow as in pumps, fans, blowers and compressors. In these cases, ASDs replace flow control valves, inlet dampers, inlet guide vanes, re-circulating throttling or on-off controls. Other benefits, such as reduced maintenance, extended motor life, more precise fluid flow control, quieter operation, etc., may also be realized, but energy saving is the principal objective.

The main characteristics of and opportunities for energy saving ASD applications are:

- In pumps, fans, blowers and most centrifugal and axial compressors, the load torque is proportional to the square of the motor speed. (Power varies as the cube of the motor speed.) Thus, reducing the motor speed by $20 \%$ will reduce the power demand by over $50 \%$.

- In most reciprocating and scroll-type compressors (mainly in refrigeration and heat-pump, applications) the load torque is constant with respect to the speed. The energy saving comes in this case from replacing on-off controls with ASDs, for continuously adjustable fluid flow.

- The control loops are closed around relatively slow-changing process variables (pressure, flow, temperature, etc.). For that reason it is necessary only that the average motor speed satisfies process needs, so that instantaneous, precise ASD speed control is normally not required and drives operate in an open loop. That fact greatly facilitates retrofits, since standard, rather than specially instrumented motors can be used. (Motor derating may still be necessary) 
- In general, these are variable torque applications with the motor power varying as the cube of the motor speed, so that no special arrangements are necessary for motor cooling at reduced speed. This also facilitates ASD retrofits.

- Both new installations and retrofits exist. However, in the case of retrofits, a range of issues (increased motor heating due to ASD harmonics, motor insulation stress, mechanical resonance, harmonics injected into the grid, etc.) must be addressed. These issues have been discussed in subsection 2.4 .

- Both low voltage (to $460 \mathrm{~V}$ ) and medium voltage $(2300 \mathrm{~V}$ to $6600 \mathrm{~V}$ ) ASDs are used. Low voltage ASDs use induction motors exclusively. Medium voltage ASDs use induction motors to 4000-6000 hp and load commutated synchronous motor ASDs above 5000-7000 hp.

- Approximately $30-50 \%$ of low-voltage ASDs have a by-pass switch, allowing direct $60 \mathrm{~Hz}$ operation, either in case of ASD failure or to improve overall efficiency with $100 \%$ load.

- There is a significant difference between low and medium voltage ASDs in terms of purchasing responsibility, technical competence of end users, maintenance and troubleshooting practices, required reliability and technical issues related to ASD interface with the motor and power grid:

-Most medium voltage ASDs are specified and purchased by an end user who can see the direct benefit of energy savings. Responsibility for the purchase of low voltage ASDs is much more diffused and can rest with an OEM, end-user or office building contractor. Hence, direct benefits of energy savings are often not included in the purchase decision.

-In general, the technical competence of end-users of medium voltage ASDs is reasonably high and certainly higher than that of low voltage ASDs users (as in commercial HVAC applications where it may be practically non-existent).

-Reliability requirements are more stringent for medium voltage ASDs, especially in the case of new installations, where mechanical means for fluid control may not have been installed, than in the case of low voltage ASDs; where pricing 
competition is greater. Yet, both low and medium voltage ASDs have to provide reliability which at least equals that of mechanical fluid flow controls.

- Digital communication is expected in both low and medium voltage drives although it may not always be used immediately. The stress here is not on communication speed (as in high performance ASDs) but on ease of installation and tune-up, on user-friendly diagnostics and functional transparency. The overall objective is to reduce the level of special training and the need for special instruments in order to use and service ASDs.

- The effectiveness of ASDs in energy saving applications is measured by the payback period, calculated as the number of years required for energy saved to pay for ASD installation. Simple payback is defined as cost of installation divided by the sum of the annual cost of energy saved and the annual savings in load demand charges, if any. It provides a quick estimate of the economics of an ASD application. A more precise result is obtained by comparing total life costs with and without the ASD or using the present value method.

The payback period may be shortened if utility rebates can be applied against the cost of ASD installation. The payback period is the most important factor governing the decision of whether to use an ASD in an energy saving application. Generally, for low voltage industrial drives, the payback period needs to be shorter than 3-3.5 years for an ASD application to be considered as economically justified. For medium voltage drives, this threshold is around 4 years. (In the case of some medium voltage ASDs, such as, for example, in forced draft fans for utility power plants, additional justification is obtained from savings realized from improvement in plant efficiency [22].)

Typically, a good candidate for ASD application would be a process requiring at least a $50 \mathrm{hp}$ motor but operating less than $20 \%$ of the time at full rated power. In addition, in the case of retrofits, an existing oversized motor would also reinforce a justification to use an ASD.

Obviously, ASD application in energy savings is much more price sensitive than in processes where speed control is mandatory. 
In summary, energy saving applications cover the power range from 10 to $30,000 \mathrm{hp}$, involving both low and medium voltage ASDs. These applications do not require high performance drives in terms of speed precision and dynamic response. Instead, the critical factors are ASD price, reliability and ease of application, above all else. If the payback period can be made shorter than 3 years, the market perceives ASD applications as economically justified.

Prospects for above average ASD growth in energy saving applications are very good since ASD market saturation is relatively low. However, in addition to ASD price, some technical issues, discussed in subsection 4.2, need to be resolved before industry's massive conversion to ASDs can happen.

\subsection{EMERGING APPLICATIONS}

This group consists of applications which do not presently use ASDs, but which could be converted to adjustable speed if the right conditions are met.

While there are many industry niches where ASDs will eventually be used, those of interest here are two very large consumer applications, both of which hold realistic prospects of using ASDs on a significant scale within this decade.

These two applications, having very diverse characteristics and ASD requirements, are the automotive and residential markets. Either of these two potential markets dwarfs, by its size, the present low voltage industrial ASD market. Assuming for a moment a very modest 20-25\% conversion to ASDs in both the home appliance and automotive markets, the resulting increase in sales volume of ASD components (such as power semiconductors, specialty chips, electrolytic capacitors, etc.) would profoundly affect the rest of the low voltage ASDs. The price of these components would be significantly reduced while ASD circuit integration would accelerate with a resulting reduction in manufacturing cost and an increase in ASD reliability.

Each of these two emerging markets is now considered. 


\subsubsection{AUTOMOTIVE APPLICATIONS}

These applications consist of using ASDs for propulsion of an electric car. Whether it is an all electric vehicle with battery or flywheel energy storage [23] or is a hybrid, electric power assisted car, an ASD would be used on board. Due to current limitations (power density, price, recharging time and life cycle) of energy storage devices, it is expected that hybrid technology will be the first to be commercialized in a significant volume.

A hybrid vehicle has an internal combustion engine, the size of which is 34 times smaller than engines used in present day cars. The engine runs at full or optimal throttle at all times (except during deceleration) to provide the base load requirement (usually defined as the power needed to move a vehicle at $50 \mathrm{mph}$ on a flat road) and to keep the batteries charged via a motor/generator and a static power converter. When driving requirements exceed the engine power output, the difference is supplied by a "battery"-static converter-motor group.

With hybrid vehicle technology there is no limit on the vehicle range, the engine emission is significantly reduced (since the engine size is reduced and since it runs full on, with almost complete combustion), the fuel economy is improved by a factor of two and the vehicle performance is not compromised.

Hybrid technology is envisaged for personal cars, utility vehicles and buses. However, several technical and manufacturing issues have to be resolved before a hybrid vehicle becomes cost competitive with the traditional technology.

Whether used with hybrid or all electric technology, the characteristics and the requirements of this application, to the extent that they have been defined, are:

- The required speed-torque characteristic is, as in any propulsion drive, very similar to that of a machine tool spindle: constant torque requirement to rated (nominal) speed and then, as wide a constant power (field weakening) speed range as possible. (Typically, the maximum speed is $300-400 \%$ of the rated speed.). The only difference between spindle and hybrid EV applications is that the propulsion drive needs to provide up to $200 \%$ torque during acceleration from standstill. 
- Motors, power converters and control will be packaged and manufactured specifically for the automotive OEMs. While the technology would be, for the most part, interchangeable with industrial ASDs, the product itself will not.

- To fit automotive applications, the drive will be torque rather than speed controlled, from zero to its maximum speed. Given the motor location, vehicle vibrations and the desired reliability, a motor shaft sensor will most likely not be acceptable and alternate methods such as speed calculation based on rotor slot current-ripple and phase-lock loop [24] will need to be perfected in order to provide overload torque capability at standstill.

- The motor voltage levels which are under consideration are in the $120-650 \mathrm{~V}$ range. At the low side, the drive can use $600 \mathrm{~V}$ IGBT switches. At the high side, it needs $1700 \mathrm{~V}$ IGBT or MCT switches, none of which are presently commercially available.

- The operating conditions (ambient temperature, shock and vibrations, overload capability and atmospheric contamination) are significantly more severe than in a typical industrial application where ASDs are often placed in air conditioned or, at least, pressurized, rooms.

- The reliability, measured in defects per million, is extremely stringent, but is helped by a relatively low number of operating hours per year.

- It is expected that a totally encased induction motor, with a projected life of 20 years, may survive the car itself to be used again in a different vehicle.

While the induction motor is presently the most serious candidate, mainly due to its cost and field weakening range, other technologies will certainly also be considered. For example, the synchronous reluctance motor may prove advantageous as operating experience accumulates [25]. (This motor type is used by one European manufacturer of spindle drives). Or, permanent magnet motors, either with sinusoidal or trapezoidal $\mathrm{mmf}$ distribution may become competitive as the price of Neodymium-Boron Iron continues to drop.

- It is estimated that, in order to reach hybrid vehicle cost parity with present car technology, a $70 \mathrm{hp} \mathrm{ASD}$, including the motor, should cost no more than $\$ 1500$. The list price of a $75 \mathrm{hp}$ general purpose industrial ASD with a motor is approximately $\$ 9,000$ to $\$ 12,000$, 
illustrating the challenge posed by the automotive application. Some of the cost reduction will be realized through volume purchasing. However, significant improvements in the design and manufacturing of ASDs is necessary before the above cost targets can be realized. At the same time, it is easy to grasp the impact that these improvements could have on industrial ASDs.

- Finally, if an "all electric' concept wins, the impact on ASD technology will be even greater:

- the required ASD rating will be closer to $100-140 \mathrm{hp}$ instead of $70 \mathrm{hp}$ as for a hybrid car

- for every electric car there will be 4-10 "battery" chargers for connecting the energy storage device to the $60 \mathrm{~Hz}$ grid. That would significantly increase the market for power semiconductors as well as some of the control chips.

\subsubsection{HOUSEHOLD APPLIANCES}

This is another huge consumer market, where ASD penetration depends primarily on economics.

Of all household applications, residential HVAC is the best prospect for ASD use. Specifically, compressor drives for heat pumps/air conditioners and central fans for furnaces, heat pumps and air conditioners may benefit most from ASD application. The reasons for this are:

- Continuous control, especially in the case of a compressor, is more efficient than an on-off control. The energy savings give a payback of 3-8 years in the case of a heat pump located in the central United States.

- Other household appliances (such as washing machines, dryers, refrigerators, mixers, whirlpool baths, etc.) either cannot derive significant improvements from ASD applications, or these improvements can be realized more economically by using motors with multi-speed windings or, most importantly, any benefit cannot justify the price premium required to pay for an ASD.

- By using ASDs to continuously control compressor and fan speeds, a continuous temperature control is obtained, providing higher comfort levels than the present on-off control. In addition, in the case of air conditioning, independent control of compressor and fan speeds allows 
for independent..control of temperature and humidity (an air conditioner can act as a dehumidifier).

Indeed, several manufacturers, of which the first was Trane, [26], have put adjustable speed heat pumps and also adjustable speed fans on the market. The response, however, was very weak, primarily because of a price premium for adjustable speed products.

At the same time, $30 \%$ of the 4 million heat pumps produced every year in Japan, are of the adjustable speed type. The difference in the acceptance of the ASD technology is explained by the difference in climates between the two countries (Japan's is generally more moderate and humid, thus amplifying the benefits of adjustable speed and favoring the use of heat pumps.), by the smaller price premium in Japan due partially to smaller heat pumps, by the difference in the cost of energy, by the difference in the marketing of adjustable speed products and by the state tax levied on low efficiency (i.e. non ASD) heat pumps.

From this discussion it is clear that the main hurdle for ASD acceptance in home HVAC applications is the price. Various economic models give the percentage of heat pump market conversion to adjustable speed as a function of the payback period. For example, it is expected that with a 1.52 year payback (1.5-2 years of energy saving to pay for the price difference between a standard and an adjustable speed heat pump) approximately $15 \%$ of the present heat pump market would convert to adjustable speed.

The second hurdle is the fact that it is not possible to retrofit a home with an adjustable speed heat pump and realize the comfort and energy benefits. Rather, the whole HVAC system needs to be changed: the thermostat, the expansion coil fan and the expansion valve, as well as the condensing coil blower. Thus, partial, gradual conversion to adjustable speed does not offer the desired benefits.

The third hurdle is the expected reliability. Traditional heat pumps last 10-15 years and those with adjustable speed cannot offer less. However, considering the extreme temperatures inside the outdoor ASD package which stress drive components, such as electrolytic capacitors, beyond their present ratings, the 10-15 ASD life cycle is presently difficult to achieve.

The fourth hurdle may be concern about harmonic distortion resulting from widespread use of residential adjustable speed heat pumps. Although 
this issue has considered by both HVAC manufacturers [27] and utilities $[28,29]$, no economic solution exists yet.

Of the two consumer applications identified in this section, the automotive one is considered more important because:

- With regulatory pressure, the probability of the conversion of present technology to ASDs is greater than in the case of household appliances.

- The potential market is larger.

- Due to the much higher cost of a vehicle (personal car, bus, etc.), the pressure on ASD prices is less severe than in the case of household appliances.

- The power rating (60-140 hp) is close to the ratings of industrial ASDs so that the technological pull-through will be more significant than in the case of household appliance drives.

- The rate of obsolescence is higher than in household applications.

In summary, this group of emerging applications offers the possibility of truly explosive ASD growth which would by far surpass the growth in existing ASD applications. The key.in realizing this opportunity is ASD cost reduction. 


\section{IMPEDIMENTS TO ASD MARKET PENETRATION}

Various issues which slow down or prevent wider use of ASDs have been mentioned earlier in this report. The objective of this section is to identify and analyze these impediments in a systematic way, to determine how they affect each of the three application groups.

Generally, impediment factors can be classified into these categories:

1. Technical: inadequate ASD performance or reliability.

2. Application: ASD installation system problems, lack of uniform standards for evaluating ASD benefits, lack of common and realistic specifications for well established applications, lack of standardized communication protocols, user-friendliness, limited availability of options with induction motors.

3. Procurement: ASD specification and purchasing channels, transparency of ASD benefits to the end user.

4. Economic: Sensitivity of market penetration to ASD price; ASD cost structure, cost of compliance with harmonic and EMI standards.

5. General: User education, legislation, consumer preferences.

Obviously, these factors are not independent since, for example, technical factors affect both applications and ASD cost. Classification is made by placing each of the issues in a category which reflects the real, not apparent, problem. For example, issues of harmonics injected into the power grid or ASD sensitivity to voltage sags are really economic/application questions, since technical solutions to overcome these problems exist.

Finally, only problems which affect ASD market penetration are considered here. For example, digital communication is not an issue since all new drives have it. However, communication protocol is, since there is no common standard permitting easy interface of drives supplied by different manufacturers.

The above factors impact in different ways each of the application groups identified in Section 3 and have a different importance for low and medium voltage ASDs. An attempt is now made to evaluate each of these factors with respect to each application. 


\subsection{APPLICATIONS WITH MANDATORY SPEED CONTROL - IMPEDIMENT FACTORS}

\subsubsection{TECHNICAL IMPEDIMENTS}

ASD technical problems may be demonstrated in lower performance or lower application reliability, or both, than desired.

Performance problems, in turn, are related to:

- Steady state precision of speed/torque control

- ASD dynamic response

- Motor problems caused by ASD supply

The performance issues in low voltage ASDs rated to $500 \mathrm{hp}$ have been essentially resolved as the technology matured, fast power semiconductors such as IGBTs became available and microprocessors accommodated complex control algorithms while permitting digital control with quick ASD tuning and easy commissioning. At the same time, circuit integration continues to reduce the part count improving reliability, control of parasitics and long term component stability. As a result, ASD performance essentially satisfies the requirements of this application group, both in motion control (servos) and high performance drives to $500 \mathrm{hp} \mathrm{[12].}$

One possible exception, mentioned only for the sake of completeness, is the case of direct drive servos, where the motor is coupled directly to a moving part, without a mechanical gear. Since torque control at zero speed is required, problems of speed resolution exist, because speed measurement and control do not benefit from a multiplying action of mechanical gears.

Finally, ASDs using IGBT devices operate with a PWM carrier frequency above $4-5 \mathrm{kHz}$ so that the current ripple is small and motor derating is usually not necessary.

While the most important performance problems have been solved, there will be further improvements with incremental benefits to ASD applications. Some examples follow:

- Elimination of shaft sensors by use of observers or MRAC (Model Reference Adaptive Control) for all but the most demanding servo applications. This will lower motor cost and increase ASD reliability. In some applications it may even be possible to use a standard induction motor with an independent cooling fan. 
- Continued replacement of DC drives with $\mathrm{AC}$ drives using high performance Field Oriented Control.

- Replacement of $\mathrm{V} / \mathrm{Hz}$ control with tachogenerator feedback by simplified (stator flux) field oriented control in single motor, packaged, ASDs. This is already happening, with the resulting benefits of improved performance, better motor utilization and elimination of shaft speed sensors.

- Development of control for operation with square-wave inverter output wave forms while retaining field orientation. This is important in all applications (such as spindle drives), where motor input frequency is increased beyond the rated value in order to attain speeds which are 3-5 times the rated one. Since the inverter output voltage, determined by the input $60 \mathrm{~Hz}$.grid voltage, cannot be increased as the frequency is increased, the motor flux is weakened, the output torque is decreased and the motor output power remains constant. Operation with a squarewave output voltage would increase the maximum attainable motor speed [30].

- Application of advanced control, such as fuzzy logic, to reduce ASD sensitivity to change in motor parameters and also to make user communication less restrictive and more friendly.

However, the above improvements are marginal and will not dramatically change the ASD application picture by opening doors to new markets.

The situation becomes quite different when one moves to ratings above $500 \mathrm{hp}$ (considered at present as the limit for the use of IGBT switches).

As one replaces IGBTs with GTO devices in PWM voltage source inverters, the carrier frequency drops by order of magnitude from $4-10 \mathrm{kHz}$ to $300-$ $800 \mathrm{~Hz}$, resulting in a substantial increase in the current ripple and, thus, increased motor heating and torque pulsations, so that motor derating or output filters become necessary. A secondary effect is that fast, high performance ASD control becomes increasingly difficult due to time delays in the current control loop, caused by slow switching GTOs. A third effect is decreased reliability and increased cost due to GTO low turn-off gain, complex gate drivers and sensitivity to voltage transients. Thus a lack of power devices with adequate characteristics for ASDs rated above $500 \mathrm{hp}$ is a definite impediment factor. 
RELIABILITY is a more important issue than performance at this stage of ASD technology development. In its broad definition, good reliability implies problem free operation. Thus the reliability covers not just statistical reliability based on component count, but also nuisance tripping, marginal operation and all design (especially software) bugs - every aspect of deviation from ASD specifications.

The reliability can be expressed either in terms of Mean Time Between Failure - MTBF (usually used by an end user or an OEM when specifying ASD reliability) or as a failure rate expressed as a percentage of the total annual production (defined by ASD manufacturers who need to worry about warranty costs and who do not know the number of ASD total operating hours, required for MTBF calculation). In terms of MTBF, the requirements are for 30,000-50,000 hours in low volume and 50,00080,000 hours in high volume ASD installations such as totally automated robot lines. In terms of failure rates. current acceptable levels have decreased to less than $0.5 \%$ during a one year warranty period, [20,31].

How does reliability affect ASD growth in this application group?

Given the fact that ASD market saturation in this group is already relatively high, significant improvements in reliability would have a limited effect on accelerated ASD growth beyond that determined by the needs of respective industries.

However, the converse is also true - if ASD reliability is not further improved, continued growth of ASD applications will slow down as the frequency of ASD caused process shut-downs increases with the increased number of installed ASDs.

An action plan for improving ASD reliability is outlined in Section 5 .

\subsubsection{APPLICATION IMPEDIMENTS}

One can find few application issues in this group which-restrict the ASD growth. The main reasons are:

- Usually well defined applications with clearly established ASD requirements, which allow manufacturers (and OEMs) to meet these requirements up front.

- Generally, relatively knowledgeable and experienced ASD buyers (normally OEMs), usually capable of correctly specifying the ASDs and 
then screening out problems during a commissioning phase before the actual production starts.

- Accumulated operating experience - there are seldom new applications in this group.

Some applications issues, however, do exist, often as a result of technological change:

- As the ASD complexity increases, the ASD-user interface sometimes becomes too complicated and difficult to use. (Examples are keypads with numerically coded commands or diagnostics, tuning procedures defined in the terms of drive rather than process variables, etc.)

- ASD sensitivity to power quality problems increase the risk of unplanned shut downs with potentially catastrophic consequences.

- As stated earlier, digital communication presently uses an RS 232 or RS 485 serial link. However, the communication protocol is not standardized - each ASD manufacturer, in fact, promotes his own in an attempt to gain a competitive advantage and also sell the "bundled" system: ASDs with PLCs (programmable logic controllers) or CNCs (computer numerical controls) and motors.

- Lack of options when selecting induction motors. DC motors, traditionally used in this application group, come with a wide range of different options, from mechanical packaging and special shafts to variety of rated speeds and voltages. Induction motors have presently

- less diversified product offerings.

- Motor leakage currents to ground and eventual motor insulation failures due to exceedingly steep voltage edges in the PWM pulse train are becoming problems in ASDs with fast switches, as explained in 2.4. The remedy for this, however, exists in the form of a common mode output filter, and/or improved motor insulation.

Although some of these issues are considered very serious, they are being addressed through improvements in ASD design, modifications in the field and general maturing of ASD industry. None of these issues is perceived to be a significant obstacle to ASD growth in this application group. 


\subsubsection{PROCUREMENT IMPEDIMENTS}

Although most ASDs are specified and purchased by OEMs rather than end-users, the ASD benefits in process control are well established and the procurement process usually reflects the application needs. As a result, procurement procedures are not seen to be a significant impediment to ASD growth.

\subsubsection{ECONOMIC IMPEDIMENTS}

While economic factors always play an important role, they are not dominant in this application group since there is relatively little elasticity in the demand for ASDs (total AC and DC drives). This means that decisions to build a steel or paper mill, to automate a production line by installing robots or to increase production capacity by adding other extruders are generally not based on the cost of ASDs. Thus, although the competition among ASD manufacturers is fierce, the total market size is basically increasing only with the growth of industries using ASDs. However, the converse is also true: a significant drop in ASD prices would decrease the cost of automation, especially in industries such as robotics and special multi-axis machining centers which use a large number of servo drives. This, in turn, would help growth in these industries and thus help ASD growth.

The only exception to the above is in the area of future conversion to ASD control in applications still using eddy current clutches, limit switches, brakes, etc., where the ASD price is very important.

\subsubsection{GENERAL IMPEDIMENTS}

Among the general factors, the most important is the need for end-users (and also some OEMs) to be informed about current developments in ASD technology. Most of the people who deal with ASDs have received their training on push-buttons, relays and M-G (motor-generator) sets and are often intimidated by ASD complexity. A new generation of plant engineers, operators and maintenance technicians, comfortable with electronic controls, software and power electronics is (very) slowly filling the industry ranks. Consequently, this is not perceived to be a serious obstacle to ASD growth, but greater availability of appropriate trade oriented training would help.

In conclusion, there are few impediments to ASD growth in the application group where speed control is mandatory. Present ASD technology tends to satisfy most of the application requirements with the exception of medium voltage ASDs. The transition from fast IGBTs to GTOs or even thyristors, as the ASD power is increased, represents a clear technological discontinuity, 
with a resulting need for motor derating, price increase, reduction in reliability and a decrease in ASD dynamic performance. While reliability of ASDs with IGBTs is presently acceptable, further improvements are necessary as the number of installed ASDs continues to increase. Finally, application, procurement and ASD pricing are not perceived as significant obstacles to ASD growth in this application group.

\subsection{APPLICATIONS WITH OPTIONAL SPEED CONTROL -IMPEDIMENT FACTORS}

The situation in this application group is very different from that in the previous one. Each of the factors is now considered.

\subsubsection{TECHNICAL IMPEDIMENTS}

While this group (and specifically energy saving applications) does not pose stringent performance requirements either in terms of speed accuracy or ASD dynamic response, the motor's efficiency is important. The presently used $\mathrm{V} / \mathrm{Hz}$ control, however, does not result in the motor's best hp/amp characteristic (the motor tends to saturate as the load is reduced due to, for example, static head change in pump and fan applications). For this reason, a simplified form of field oriented control (for example, based on stator flux, [16]) will most likely be used in the next generation of ASDs. While this may improve motor efficiency, this change is not viewed as critical for future ASD growth.

ASD reliability needs to be improved so that it becomes at least comparable to that of the mechanical devices which ASDs are replacing. Specifically, what is needed is to remove a range of nuisance ASD problems related to marginal design, component reliability, application factors and user interface which infuriate and discourage non-expert end users and which continue to give a reputation of poor reliability to ASDs. In fact, field data in energy saving applications show that once initial installation and start-up problems are solved, ASDs tend to offer very good reliability, [22,32], (most likely, at least partially, due to decreased ASD stress with variable torque loads). This also points to a need for a better application approach and end-user knowledge. Some steps for improving ASD reliability are outlined in Section 5 .

The most important technical issue, however, is the lack of a suitable . power switch for drives rated above $500 \mathrm{hp}$. In all voltage source inverters above 400-500 hp, GTOs are used with a resulting increase in motor current ripple, an increase in motor losses and a need for motor derating at full power. At still higher power, current source inverters, used in medium voltage ASDs, pose a different type of problem, more related to ASD cost 
than performance. In both cases, the availability of a suitable power switch, with characteristics comparable to an IGBT or even a bipolar transistor would increase ASD applications in the higher power ranges.

\subsubsection{APPLICATIONS IMPEDIMENTS}

Possibly due to the versatility of use, application factors can significantly influence ASD growth in this application group. The most important factors are:

- ASDs, especially in the low voltage (20-100 hp) range, are applied and often specified by people having very vague notions about ASD characteristics. The ASD is often inserted between the grid and motor with no disconnects, no protection coordination with other equipment and no thought given to ASD sensitivity to power quality problems. Often, the results are application problems with severe disappointments and consequent discredit of ASDs.

- The commercial HVAC industry appears confused by ASD manufacturers' claims about ASD efficiency and energy saving potential. These claims are often exaggerated and have become a sales tool. When the claims are not realized, there is a backlash with a loss of ASD credibility. What is needed is a clear ASD evaluation procedure that will become a standard for bench marking everybody's equipment [33]. Also needed is users' awareness of how to compute energy savings for a given application.

- In some applications, such as commercial HVAC, the ASD specifications often exceed the application requirements. This is primarily a consequence of the "specsmanship" game, where some manufacturers promote features not related to the application needs in an attempt to gain a competitive advantage. When these features become incorporated into ASD specifications, competition becomes limited and ASD price increases. What is needed are standardized, core specifications for most common applications [34].

- Digital communication protocols now vary from manufacturer to manufacturer causing compatibility problems, especially with retrofits. The protocols should be standardized and agreed upon.

- Given the relatively limited technical knowledge of most end users in this application group, ASD-user interface has to become more meaningful to the application. For example, the settings should not be in terms of voltage boost, $\mathrm{V} / \mathrm{Hz}$ curves, current limits, etc., but related to 
the starting torque, time to full speed, maximum torque, etc. The responsibility for user-friendly communications rests primarily with ASD manufacturers.

On the up-side, having a microprocessor on board, one can turn an ASD into an intelligent control center, which can do much more than just control speed. That approach has been partially taken in the past - some examples of auxiliary functions implemented in general purpose drives are:

- Software PI (proportional-integral) controller for closing process loops such as temperature, pressure, flow, etc. controls.

- Speed jump - drive avoids continuous operation at pre-selected speeds so as to avoid mechanical resonance.

- Master/slave connection, creating an electrical shaft with selectable "gear ratio."

- Automatic inspection and diagnostics of motor damage (such as broken rotor bars in induction motors).

- Energy saving: reduced motor flux at light loads.

- Automatic braking by DC currents immediately before each start-up.

- Automatic reset and restart after the power has been restored following short outages.

- User-friendly dialog, implemented through keypad/display units, which guides user through set-up, commissioning and diagnostics.

In the future, ASD growth can be enhanced by leveraging microprocessor availability to perform additional control tasks.

\subsubsection{PROCUREMENT IMPEDIMENT}

Procurement procedures definitely impede ASD sales in low voltage, 30$200 \mathrm{hp}$ energy saving applications. For example, in cases of commercial HVAC, a contractor who selects electrical equipment has no incentive to increase his cost by specifying energy saving ASDs and the user is not knowledgeable enough to insist on them. 
In industrial energy saving applications, the selection of electrical equipment is normally decided on by a plant manager, whose first responsibility is to keep the plant operating. A previous reputation for low reliability adversely affects the choice of an ASD.

The procurement picture is better for medium voltage, high power ASDs with applications in power plants, waste water treatment stations and pulp and paper industries, where ASD energy saving benefits are recognized and large power ratings (and thus energy savings) result in shorter paybacks.

Utility rebates are a positive factor throughout the industry; their impact is greatest if the rebates are available to end users directly, rather than through Energy Service Companies.

\subsubsection{ECONOMIC IMPEDIMENTS}

ASD price directly affects an ASD purchase decision since the choice of an ASD is compared with other options. In this respect, and unlike the previous application group, the market is very elastic and the rate of ASD growth depends almost entirely on ASD price. In fact, ASD price is the single most important factor determining ASD market penetration in this application group. The cost issue will become even more important when mandated standards for reduced harmonic distortion become enforced. Compliance with these standards will cause an increase in ASD prices and may set back ASD growth.

The opportunity for ASD cost reduction exists with both low voltage and medium voltage drives. Specific actions are outlined in section 5 .

\subsubsection{GENERAL IMPEDIMENTS}

The most important factor in this category is end-user education and the resulting perception of ASD benefits, reliability and system application issues. Each of these topics has to be clearly presented to the end-user community by an organization which is perceived to be objective and which has no direct stake in increasing ASD sales.

Finally, one can view the low price of energy in the U.S. as another impediment to ASD growth.

Again, some actions concerning this issues are outlined in section 5. 


\subsection{EMERGING APPLICATIONS - IMPEDIMENT FACTORS}

While the full picture of all requirements in emerging markets is not yet formed, it is clear that ASD price and implied reliability are the two dominant factors determining market conversion to ASDs. As stated in Section 3, the price at which an ASD is economically competitive with the present technology in a hybrid-electric vehicle (EV) application is approximately $15-20 \%$ of the present listed price of industrial ASDs. The price pressure is even greater in residential HVAC applications. Although some applications, such as the electric car, may be legislated, significant cost reductions are still needed before ASDs become a serious factor in consumer markets. Several proposals on how to address the cost are given in Section 5.

While cost is the most dominant factor when one starts considering ASDs in consumer applications, the required reliability is most often implied. Yet, current ASD reliability would not meet the requirements of these new applications, at least not to end-user satisfaction. This means that while the current technology can provide adequate ASD protection and survivability, the number of nuisance trips, service calls, sensitivity to disturbances and just the life of some of the components would result in consumer disappointment. For that reason, some actions to improve the reliability are proposed in Section 5.

Other factors, which are not decisive to ASD penetration into emerging applications, but which could be an issue if the cost and reliability become more acceptable are:

Acoustic noise. This is an important factor in both residential and EV applications. The use of inverters with ultrasonic switching (carrier frequency above $18-20 \mathrm{kHz}$ ) either in hard switching or resonant configurations still represents a cost penalty. Even if 4th. and 5th. generations of IGBTs reduce this penalty, the problems of EMI containment and motor insulation stress will remain with very high frequency switching. The soft switching techniques would remove these problems, but the cost premium for added resonant circuits remains.

Sensorless control. This is an issue in EV and possibly in heat-pump (hermetic compressor) applications, when the motor has to develop 100$150 \%$ of rated torque at starting. Neither application accepts motor shaft sensors, so that rotor position/speed need to be calculated from electric measurements with sufficient accuracy, at very low speeds and starting from standstill. While several methods to this effect have been proposed 
(third harmonic sensing, rotor slot harmonics sensing with phase locked loop [24], etc.) none has yet been implemented in an industrial product.

Maximum speed. This is the same issue discussed in 4.1 .1 (spindle application) and deals with a maximum attainable speed for a given supply voltage. (Motor control with an acceptable performance with square-wave voltage supply at high speeds). Due to market size, the issue is even more important in EV applications.

Heat transfer. Applications such as residential heat pumps have used up to now (in Japan and the U.S.) only natural, convection cooling because of reliability concerns with cooling fans and clogged filters and overall efficiency loss in case of freon cooling. In EV applications, the drive will most likely need to be liquid cooled, again due to concerns about air filter maintenance, drive weight and size, and sealing electronics from outside contaminants.

In summary, ASD price and reliability are the two key issues that have to be significantly improved before large scale use of ASDs in emerging consumer markets can become a reality. Some other factors which may become important as emerging applications materialize have also been identified. 


\section{ACTION PLAN}

This section presents key actions, which, if carried out, would lead to better and more widely accepted applications of ASDs. The recommendations are divided into two groups. The first presents actions which could yield results over the next 5-6 years; in the second, the results would be obtained after 10-20 years. It is understood that the responsibility for implementing these recommendations is diffused and that not all actions may be carried out.

Each of the two groups is now considered.

\subsection{SHORT TERM BENEFITS}

The results of the actions identified here would be obtained by the year 2000 if the actions were implemented soon. Each recommended action is listed in each of the categories which it impacts.

\subsubsection{COST REDUCTION}

1. Develop a comprehensive plan, on the national and possibly international level, for resolving the issue of ASD generated line harmonics. These issues will become increasingly important as the number of installed ASDs continues to grow and as the proposed standards threaten to increase ASD cost.

Some of the questions are: should each ASD have to satisfy harmonic and power factor norms, as presently required by IEC - 555 ? That would unduly penalize ASD cost in applications where ASDs represent a small portion of the total load. Should each user have to comply with limits on harmonic distortion, measured at his point of common coupling (customer service entrance), as specified by IEEE 519? How will this standard be enforced? Would the required expertise make ASDs specialty equipment, further limiting their applications? Finally, is it more economical, on a national level, to prevent harmonics from being generated (by, for example, using PWM controlled rectifiers) or is it better to use minimum cost ASD topology (that is, a diode rectifier) and have large, active filtering

- stations to keep the harmonics at an acceptable level?

Optimal resolution of these issues requires technical leadership at the national level which neither ASD manufacturers nor users can provide. 
The outcome of the standards-harmonics debate will impact not only ASD domestic growth but also the international competitiveness of US manufacturers.

2. Foster development of standard specifications for the most common ASD applications such as commercial HVAC. The objective would be to facilitate procurement and to increase the competition by making bidding easier against a well defined, clear set of requirements. This is especially relevant in applications where people specifying ASDs do not have the necessary technical background and are often swayed by overly cautious consultants or by sales claims of some manufacturers with respect to features of their own ASDs.

3. Foster development of a common protocol for serial link communication. As stated earlier, the communication hardware presently uses RS-232 and RS-485 standards, but there is no communication software standard. The objectives would be two-fold:

- Lower the cost by facilitating applications and increasing competition among ASD vendors by permitting a mix-and -match of various drives, programmable logic controllers (PLCs) and other industrial controllers.

- Improve, if possible, the user friendliness by making ASD commissioning and use easier and more transparent than they are presently. That, in turn, would reduce the need for special ASD user training.

Once the standards are established, the entire communication software package could be eventually supplied by third party software houses (as it is presently the case in the personal computing industry) to further dramatically reduce ASD cost and increase ease of use.

4. Foster development of common integrated circuits which would be available to all domestic ASD manufacturers and which would:

- Reduce the cost of developing an ASD product by having the part already available

- Reduce ASD cost by using a high volume chip to achieve circuit integration 
- Increase reliability by reducing part count and especially by eliminating opportunities for design errors.

For example, a chip to implement Direct Field Oriented Control, with no shaft sensors, would be used in a wide range of applications while realizing all the benefits listed above. (A similar project is going on in Europe, supported by, among others, ABB, Siemens, AEG, Bosch, Danfoss, Indramat, Lenze, Moog, Reliance, and SEW, to develop a Ve-Con (vector control) chip for high performance applications. The chip proposed here would be aimed at high volume applications, as a replacement for $\mathrm{V} / \mathrm{Hz}$ control.)

Another example would be a standard communication chip implementing the kernel of the communication protocol in hardware. Similar chips, supporting different protocols already exist: Interbus$S$, Lanworks and CAN. If a standard chip were available, it would reduce the cost and improve the reliability of ASDs.

Any other part of ASD circuitry, such as, for example, the power supply, could also be integrated into a chip. The philosophy is the same as the one which was so successful for circuit integration in signal and consumer electronics:

- Reduce the cost of product development by doing the design only once, in the chip, instead of repeating it every time someone wants to develop that product.

- Improve product performance and reliability by bringing the best designers to the task.

- Increase the reliability by reducing the part count.

- Ultimately reduce the product price as increased volume drives chip price down.

Globally, these efforts do not have to be restricted to circuits suitable for monolithic integration. By having entire ASD subsystems standardized, it would be possible to create ASD or Power Electronics Building Blocks (PEBB), such as control board, IGBT driver and logic power supply boards, etc. The existence of these PEBB would further reduce ASD costs while shortening the new product design cycle. 
5. Foster research into ASD topologies having a potential for reduced cost.

The first project would be to develop a working model of an ASD without the DC link filter. The prototype would be tested under various load conditions and AC power supply imbalance to determine the limitations of such a topology. Elimination of the DC link filter would considerably reduce ASD cost (by eliminating both the filter and the DC capacitor pre-charging circuit), extend ASD life and improve the ASD input power factor. While eliminating the DC link filter has been proposed in the past, $[35,36]$, there is no data available regarding the practical limits of such a topology.

The second project would be to expand on the recent work concerning single-to-three phase converters [37] and to explore alternate topologies for low cost drives with a reduced number of solid-state switches.

6. Foster development of low cost motors for residential HVAC and possibly EV applications.

While the induction motor will continue to dominate industrial applications rated $5-5000 \mathrm{hp}$, new consumer applications with no replacement compatibility constraints and with highly integrated. OEM products may benefit from other motor technologies. Specifically, surface mounted permanent magnet (PM) motors with a trapezoidal mmf may be attractive because:

- Their efficiency is essentially constant over the entire load range while that of an induction motor drops off significantly as the input current decreases, approaching the motor magnetizing current.

- They require a $15 \%$ lower voltage rating of power switches than motors requiring a sinusoidal supply. (For the same RMS value, a trapezoid has $15 \%$ lower peak than a sinusoidal wave form). However, this advantage may be realized only if custom switches are used since the commercial switches are rated at 600 and $1200 \mathrm{~V}$. 
Other motor concepts which may hold a promise of reduced costs compared to an induction motor are:

- synchronous reluctance motor [25].

- permanent magnet assisted, switched reluctance motor.

- interior (buried) magnet motors in conjunction with appropriate manufacturing methodology, etc.

If such projects were undertaken, it would be important to focus them on the low cost objective, using an equivalent induction motor as a bench mark.

7. Fostering development of low cost magnets which could be used in PM motors. A significant reduction in the cost of Neodymium-BoronIron magnets would drastically change the cost equation, permitting economical use of PM motors in high volume consumer applications. In the process, motor cost and size would be reduced. For this, however, to happen, the present cost of permanent magnets has to be reduced.

8 Fostering development of manufacturing technology of PM motors. At present, a large portion of the motor manufacturing cost is related to the attachment of magnets to the rotor surface. The operation is performed manually and offers an opportunity for significant cost saving in the future.

9. Fostering development of low cost ASD manufacturing technology. This project would involve an assessment of the potential for cost reduction in the manufacturing cycle of present day ASDs, including both manufacturing and ASD testing, followed by identification of possible improvements.

\subsubsection{RELIABILITY IMPROVEMENT}

1. Promote circuit integration by fostering development of various, strategic high volume ASD chips which would:

-facilitate ASD design, thus reducing the possibility of design errors

-reduce the ASD part count. 
Details of these recommendations may be found in the preceding sub-section (Cost Reduction).

2. Publish "Good Engineering Practice" ASD design guidelines including recommended voltage and current derating of each component as a function of desired MTBF. The guidelines should also address designs for manufacturability and for compliance with safety standards.

3. Publish recommended ASD qualification procedures for most common applications. The procures would outline the tests which an ASD prototype should pass before it is released to production. The tests would address both design integrity and compliance with the existing standards.

4. Develop a dependable methodology to evaluate ASD reliability at the design stage and to track the effect which design changes (such as those related to cost reduction) have on the reliability.

Such procedures exist for evaluating the reliability of military equipment but are not applicable to industrial, commercial or residential-ASDs. Yet, the ability to predict ASD reliability with a high degree of certainty is crucial when very high volume consumer applications are considered.

5. Foster development of ASD topologies and circuitry which would not contain the parts with lowest reliability: electrolytic capacitors, optocouplers, interconnects (such as plug-in connectors), etc.

6. Foster development of technologies and methods for improved heat transfer and heat management. The operating temperature directly affects component failure rates. At the same time, the effectiveness of heat removal affects the ASD cost. The issue of heat management is especially important in very large drives and in applications (such as, for example, residential heat pumps) where reliability concerns do not allow use of conventional cooling fans.

\subsubsection{PERFORMANCE IMPROVEMENT}

1. Foster development of economical methods for reduction of acoustic noise generated by ASD operation. That involves:

- A search for enclosure materials which would dampen specific ASD frequencies. 
- Identification of noise generating components such as, tantalum capacitors under some operating conditions, and recommendation of replacement alternatives.

- Development of PWM control to distribute the acoustic energy over the frequency spectrum.

- Any other approach to reduce the noise without increasing ASD cost.

2. Foster development of control techniques which would permit an increase of motor maximum speeds for a given supply voltage in field oriented ASDs. At present, a vector controlled ASD never uses full available voltage across the DC link during motor field weakening in order to keep the current control loop out of saturation.

Ideally, it would be possible to go into operation with square-wave output voltage (which is the maximum voltage available), temporarily losing field orientation, which is not important at maximum motor speeds. The development would focus on how to pick-up the field orientation again, as the motor speed is reduced. This issue is of concern to spindle and EV applications.

3 Foster development and demonstration of the methods to develop high torque (say, $200 \%$ of the rated torque) during acceleration from standstill with ASDs having no position or speed sensors on the motor shaft.

The issue is of concern to EV applications where use of sensorless induction motors is preferable.

4. Foster assessment and possible development of resonant topologies for high power ASDs.

A resonant (or DC side auxiliary commutated) inverter has been promoted over the last seven years with promises of reduced cost [38]. When these promises could not be realized, the concept lost credibility in industry. Its commercialization failure was caused primarily because resonant switching was aiming to replace hard switching of efficient and fast IGBTs. In that case, the potential benefits are more than offset by the cost of the added resonant circuit and increased control complexity. If, instead, the concept were applied to GTO inverters, it could increase GTO switching frequency, thus reducing the current ripple and the motor losses. In this way, the savings realized by not derating the motor and by reducing the motor losses might be sufficient to pay for the cost of the resonant 
circuit. Another new resonant circuit [39] may achieve these objectives and offer all the advantages of soft switching at lower cost than the circuit prop[proposed in [38]

\subsubsection{USER EDUCATION AND GENERAL FACTORS}

1. Consider guidelines which would determine minimum efficiency levels in commercial HVAC, municipal water treatment and industrial air handling.

2. Promote end-user education, especially in commercial HVAC, about the benefits and correct application of ASDs. The application information is particularly needed in cases of ASD retrofits, where the end user has lived for years with a constant speed motor.

\subsection{LONG TERM BENEFITS}

The benefits from the actions recommended here would be available in 10-20 years. Given the time span, one could speculate about a range of actions which may or may not turn out to be beneficial in the end. For this reason, only two actions which would result in specific and much needed ASD improvements are recommended.

\subsubsection{DEVELOPMENT OF NEW POWER SWITCHES}

As stated in Section 2, there is a technological discontinuity between ASDs using IGBTs and ASDs using GTOs or thyristors. Single IGBT devices allow ASD ratings to approximately $250-300 \mathrm{hp}$, depending on the required overload capability. Paralleling IGBTs extends the power range to 500-600 hp. Above that power level one has to use GTOs or thyristors in various voltage or current source ASD configurations, with a resulting drop in ASD performance and reliability and an increase in cost.

The first objective in this proposed project would be to develop an efficient static power switch of a rating comparable to those of current GTOs $(6 \mathrm{kV}$ and $4 \mathrm{kA}$ ) and with switching characteristics (speed, gate power and onstate voltage drop) comparable to those of an IGBT. The availability of such a switch would significantly energize the large future market of medium voltage ASDs in energy saving applications. The development of such a switch may be based on a MOS-Controlled-Thyristor (MCT), currently being developed by Harris Corporation [40], on plasma switching techniques, or may start from scratch. 
A second objective would be to develop a bi-directional, gate controlled switch, ideally with switching characteristics comparable to those of an IGBT. Such a switch would revolutionize the field of power electronics by making so-called Matrix Converters [41] (or Direct AC-AC converters) economically feasible. The benefits would be elimination of the DC link, elimination of the input and output low order harmonics, operation with a unity power factor and the ability for a bi-directional power flow (motoring and regeneration to $\mathrm{AC}$ lines.)

The third objective would be to explore the replacement of silicon in power switches by new materials, such as gallium-arsenide (GaAs) or siliconcarbide ( $\mathrm{SiC}$ ). The main benefits would be a more than doubling of the permissible junction temperature and a substantial increase in switching speeds. However, such a development would really be of a long term nature.

Finally as a closure to the topic on power semiconductors, one should note that the main suppliers of both IGBTs and GTOs are either in Japan or Europe. It is generally considered that the party holding power semiconductor technology also controls the cost and technological future of ASDs. The survival of the remaining U.S. manufacturers of ASDs and above everything, their leadership role, is problematic without strong domestic power semiconductor suppliers.

\subsubsection{APPLICATION OF SUPERCONDUCTIVITY TO ASDs}

As already stated, thermal management represents a serious challenge at high power levels for both motors and static converters. The present advancements in superconductivity technology could not only alleviate the thermal management problem but, more importantly, substantially increase ASD reliability by lowering the operating temperature and, in the process, also improving the motor-converter efficiency.

In fact, although this recommendation is considered to promise long term benefits, a demonstration of the advantages of a superconductive ASD could be expected before the end of this decade. This is particularly true for the case of a DC choke in medium voltage ASDs, which accounts for most of ASD losses and which would be the first candidate for application of superconductivity. 


\section{CONCLUSION}

In this report, present and potential ASD applications have been divided into three large groups:

1. Processes where speed control is part of a process technological requirement. In the past, speed control in this application group may have been achieved through mechanical, hydraulic or electromechanical (motor-generator) means. However, presently, almost all applications in this group already use ASDs. In fact, ASD growth in the 1970-1985 period was generated primarily by conversion from other methods to electronic speed control. Although most of these applications would welcome improvements in some ASD characteristics and certainly a decrease in ASD prices, there is no single impediment, the removal of which would result in a significant ASD growth in this market. The market demand is perceived to have relatively little elasticity and ASD growth in this application group will essentially track the respective industry growth. Some small differences may be caused by conversion to electronic speed control in the last remaining applications where eddy-current clutch, hydraulic or clutch-brake-limit switch operations are still used.

However, the converse is also true: a significant drop in ASD prices, caused, for example, by very high volume applications in other markets, would lower the overall cost of process machinery which use ASDs and would thus be an agent of industry growth.

2. Processes which can benefit from speed control but where adjustable speed is not mandatory. Of all applications in this group, the most important one is in energy savings, involving pumps, fans and compressors (PFC).

The most important factors affecting ASD growth in this group are ASD price, reliability, cost of energy and, in the 20-200 hp range, ease of application, end-user education and user friendliness. Since ASDs are competing with other solutions (such as valves, dampers, vanes, etc.) primarily on an economic basis, this market has considerable elasticity to ASD growth, primarily depending on ASD cost.

This group offers the best opportunity for above average ASD growth over the next 5-6 years, providing that current levels of ASD price, ASD reliability (real or perceived) and user education are improved. 
The opportunity here lies in the fact that the required improvements are not dramatic and are realistically possible. A specific action plan to bring about these improvements was outlined in Section 5.

3. Emerging applications, where ASDs are not presently used, but could be used if the required cost and reliability levels are met. The most important emerging applications are in large consumer markets, specifically in Hybrid Electric or all Electric Vehicles (EVs) and residential heating, ventilation, and air conditioning (HVAC). By their potential size, these applications dwarf the total current ASD sales volume. If these emerging applications were realized, the resulting changes in ASD cost structure, reliability and manufacturing technology would also have a very large impact on all other low voltage ASD applications. In fact, the ASD manufacturers who succeed in satisfying the cost and reliability requirements of EV applications will most likely evolve to dominate all other ASD markets.

However, although use of ASDs in EVs is helped by clean air legislation, substantial reduction in ASD cost and improvement in ASD reliability are needed before ASD powered cars can compete with the current technology.

The conditions for ASD penetration into the residential HVAC market are even more stringent, especially with respect to ASD cost.

Again, an action plan to reach the required cost and reliability levels was outlined in Section 5 .

In summary, the opportunities for significant ASD growth lie in applications where ASDs are competing practically entirely on price with other technologies. These applications do not normally need better ASD performance, beyond what is available today, but require significant ASD cost reduction from present levels. Thus, any ASD technological improvement which carries a cost penalty may help penetrate a specialized, small niche, but is not the right improvement to bring high volume ASD applications closer to reality. Therefore, future $R$ \& $D$ efforts should concentrate on reducing ASD cost and improving ASD reliability and ease of use, in order to realize the ASD growth potential in energy savings and consumer applications. 


\section{REFERENCES}

[1] P.M. Bhagwat and V.R. Stefanovic: "Generalized Structure of a Multilevel PWM Inverter", IEEE-IA Trans., Vol. IA-19, No.6, Nov/Dec. 1983, pp. 1057-1069

.. [2] A. Nabae, I. Takahashi and H. Akagi: "A New Neutral-Point-Clamped PWM Inverter" IEEE-IA Trans., Vol. IA-17, No.5, Sept/Oct. 1981, pp. $518-523$

[3] T. Salzmann, G. Kratz and C. Daeubler: "High-Power Drive System with Advanced Power Circuit and Improved Digital Control", IEEE-IA Trans., Vol. 29, No.1, Jan/Feb. 1993, pp. 168-174

[4] A.H. Mittag: "Electric Valve Converting Apparatus", U.S. Patent $1,946,292 ; 1934$

[5] C.C. Rettig: U.S. Patents $3,863,121$ and $3,863,122 ; 1965$, and U.S. Patent 3,962,614; 1976

[6] K.P. Phillips: "Current Source Converter for AC Motor Drives", IEEE-IA Trans., Vol. 8, No.6, Nov/Dec. 1972, pp. 483-488

[7] Ansaldo-Hill Graham Controls Ltd.: Description of Operation of IMVAR Drive, July 1990

[8] E.P. Wiechmann, P.D. Ziogas and V.R. Stefanovic: "A Novel Bilateral Power Conversion Scheme for Variable Frequency Static Power Supplies", IEEE-IA Trans., Vol. IA-21, No.5, Sept/Oct. 1985, pp. $1226-1233$

[9] M. Hombu et al: "A New Current Source GTO Inverter with Sinusoidal - Output Voltage and Current", IEEE-IA Trans., Vol. 21, No.5, Sept/Oct 1985, pp. $1192-1198$

[10] P. Espelage et al: "Symmetrical GTO Current Source Inverter for Wide Speed Range Control of 2300 to 4160 Volt, 350 to $7000 \mathrm{hp}$ Induction Motors", Conf. Record, 1988 IEEE-IAS Annual Meeting, pp. 302-307

[11] N. Kleinsorge, U. Putz and H. Stemmler: "Large Adjustable Speed AC Drives", Conf. Proceedings, 3rd European Conference on Power Electronics and Applications, Aachen 1989, Vol. 1, pp. 491-498 
[12] V.R. Stefanovic: "Industrial AC Drives-Status of Technology", EPE Journal, Vol. 2, No.1, March 1992, pp. 7-24

[13] P. Vas: Vector Control of AC Machines, Oxford University Press, 1990

[14] A.M. Trzynadlowski: The Field Orientation Principle in Control of Induction Motors, Kluwer Academic Publishers, 1994

[15] R.W. DeDoncker and D.W. Novotny: "The Universal Field Oriented Controllers", Conf. Record, 1988 IEEE-IAS Annual Meeting, pp. 649-656

[16] X. Xu, R.W. DeDoncker and D.W. Novotny: "A Stator Flux Oriented Induction Machine Drive", IEEE-PESC, Kyoto 1988, pp. 870-876

[17] H. Schierling: "Self-Commissioning: A Novel Feature of Modern Inverter-Fed Induction Motor Drives", Proc. of 1988 IEE Conf. on Power Electronics and Variable Speed Drives

[18] E. Persson: "Transient Effects in Application of PWM Inverters to Induction Motors", IEEE-IA Trans., Vol. 28, No.5, Sept/Oct. 1992, pp. 1095-1101

[19] Y. Murai et al: "Leakage .Current Reduction for a High-Frequency Carrier Inverter Feeding an Induction Motor", IEEE-IA Trans., Vol. 28, No.4, July/Aug. 1992, pp. 858-863

[20] Private Communication with M. Dudzinski, CEO, American Industry Controls, Charlotte, NC

[21] Ducker Research 'Co.: "Report on Adjustable Speed Drive Market and Applications", Nov. 1992, performed for EPRI

[22] J.A. Oliver and A.A. Eslami: "Retrofitting Utility Power Plant Motors for Adjustable Speed: Field Test Program", Report on Projects RP1966-6 and RP.2951-6, EPRI, 1990

[23] Proceedings of Flywheel Energy Storage Technology Workshop, Oak Ridge National Laboratory, November 1993 
[24] D.S. Zinger et al: "Using Induction Motor Stator Windings to Extract Speed Information", Conf. Record, IEEE-IAS 1989 Ann. Meeting, pp. $213-218$

[25] T.A. Lipo: "Synchronous Reluctance Machines - a Viable Alternative for AC Drives?", Journal on Electric Machines and Power Systems, 19: 659-671, 1991

[26] Product Literature, Trane XV1500 Variable Speed Air Conditioner

[27] L. Sulfstede: "A Residential Single Phase Variable Speed Heat Pump or Air Conditioner with High Power Quality and Low Harmonic Currents", Proceedings of Second International Conference on Power Quality, Atlanta, GA, September 1992, pp. C-16-1 to C-16-8

[28] K.J. Olejniczak et al: "An Evaluation of the Harmonic Impact of Residential Variable Speed High Efficiency Heat Pumps", Proceedings of Second International Conference on Power Quality, Atlanta, GA, September 1992, pp. C-15-1 to C-15-8

[29] W.M. Grady et al: "Field Measurements and Preliminary Study Of Harmonic Distortion Caused by Distributed Single-Phase ASD Heat Pumps", Proceedings of Second International Conference on Power Quality, Atlanta, GA, September 1992, pp. B-13:1 to B-13:5

[30] J.Holtz et al: "On Continuos Control of PWM Inverters in the Overmodulation Range, Including the Six-Step Mode", IEEE-PES Trans, Vol. 8, No.4, October 1993, pp. 546-553

[31] Private Communications with F. Mellon, President, Melltronics, Inc., Grand Island, NY

[32] D.C. Azbill, R.E. Catlet and J.E. Propst: "A Case Study of Replacing Steam Turbines with LCI Type Variable Speed Drives", Conf. Record, IEEE-IAS 1990 Annual Meeting, pp. 1438-1445

[33] Private Communications with R. Orndorf and M. Liptak, Square D Co., Raleigh, NC

[34] Private Communications with J. Reichard, Division Director, Reliable Electric Controls (Metropolitan Pump Co.), Brookfield, WI 
[35] P.D. Ziogas, Y.G. Kang and V.R. Stefanovic: "Rectified-Inverter Frequency Changers with Suppressed DC Link Components", IEEE-IA Trans., Nov/Dec. 1986, pp. 1073-1081

[36] I. Takahashi and Y. Itoh: "Electrolytic Capacitor-less PWM Inverter", IPEC - Tokyo, 1990, pp. 1311-138

[37] P.N. Enjeti and A. Rahman: "A New Single-Phase to Three-Phase Converter with Active Input Current Shaping for Low Cost AC Motor Drives", IEEE-IA Trans., Vol. 29, No.4, July/Aug. 1993, pp. 806-812

[38] D.M. Divan and G. Skibinski: "Zero Switching Loss Inverters for High Power Application", IEEE-IAS 1989 Meeting, pp. 627-635

[39] J.S. Lai: "Resonant Snubber Based Soft Switching Inverter" Invention Disclossssure No.1430-X, Energy Systems Division, Oak Ridge National Laboratory

[40] MCT User's Guide, Harris Semiconductors, September 1992

[41] M. Venturini: "A New Sine Wave In, Sine Wave Out Conversion Technique Eliminates Reactive Components", Proc. PowerCon 7, 1980, pp. E3-1 to E3-15 


\section{DISTRIBUTION}

ORNL/Sub/80-SN772

\section{Internal}

1. Curt Ayers

2. Van Baxter

3. Don Casada

4. F. C. Chen

5. Ned Clapp, Jr.

6. G. E. Courville

7. Walter Dykas

8. P.D. Fairchild

9. Uri Gat

10. Dr. S. G. Hildebrand

11. Ed Hillsman

12. Patrick Hughes

13. John Hsu

14. P.A. Jallouk

15. N. Jett

16. John Kueck

17. J.M. Macdonald
18. Howard McClain

19-26. Ben McConnell

27. V.C. Mei

28. C. I. Mosers

29. R. W. Murphy

30-50. M. Olszewski

51. Pedro Otaduy

52. C.K. Rice

53. R. M. Schilling

54. Terry Sharp

55. R. B. Shelton

56. John Stovall

57. J. VanCoevering

58. Brian West

59. ORNL Patent Office

60. Central Research Library

61. Document Reference Section

62-63. Laboratory Records ( 2 copies)

64. Laboratory Records RC

\section{External}

65. Harry R. Baker, President and CEO, Marathon Electric, Wausau, WI 54402

66-70. Rich Been, Energy Engineer, U.S. Department of Energy/Seattle, 800 Fifth Ave., Suite 3950, Seattle, Wasshington 98104

71. Tom E. Bettcher, President, Copeland Corporation, 1675, West Campbell Rd, Sidney, OH 43365-0669

72. Stan Blazewicz, U.S. Department of Energy, 5G-087 Forrestal Bldg, EE-221, 1000 Independence Ave., SW, Washington, DC 20585

70-75. Chris Cockrill, U.S. Department of Energy, 911 Walnut, 14th Floor, Kansas City, MO 641062024

76. Dr. Thomas E. Drabek, Professor, Department of Sociology, University of Denver, Denver Colorado 80208-0209

77. Mark Dudzinski, CEO, American Industrial Controls, 2744 Yorkmont Road, Charlotte, NC 28208 
78. David O. Gifford, President, Emerson Motor Company, 8100 West Florissant Ave., P.O. Box 3946, St. Louis, MO 63136

79. Linos Jacovides, Head, Electrical Engineering Department, General Motors Research Laboratories, 30500 Mound Road, Warren, MI 48090-9055

80. Tom M. Jahns, Corporate R\&D, General Electric Company, P.O. Box 8, Room EP-101, Schenectady, NY 12301

81. Michael Liptak, Electronic Product Development, Square D Company, P.O. Box 27446, Raleigh, NC 27611

82. John M. Miller, Vehicle Electronics Department, Ford Research Laboratory, 20000 Rotunda Drive, P.O. Box 2053, MD-2036, Dearborn, MI 48121-2053

83. Tom A. Nondahl, Standard Drives Division, Allen-Bradley Co., 6400 West, Enterprise Drive, Mequon, WI 53092-0760

84. Jeff A. Reichard, Division Director, Reliable Electric Controls, 4465 N 124th Street, Unit C, Brookfield, WI 53005

85-90. Paul E. Scheihing, U.S. Department of Energy, 5G-087 Forrestal Bldg, EE-221, 1000 Independence Ave., SW, Washington, DC 20585

91. Burley Semones, Vice President, Engineering, Industrial Drives-Kollmorgen, 201 Rock Road, Radford, VA 24141-4099

92. Mark E. Shepard, Engineering Center, Otis Elevator Co., 5 Farm Springs, Farmington, CT 06032

93. George F. Sowers, Senior Vice President, Law Companies Group, Inc. 114 Townpark Drive, Suite 250, Kennesaw, GA 30144-5599

94-103. Victor R. Stefanovic, Box 333, Rt. 2, Afton, VA 22920

104. Dr. C. Michael Walton, Ernest H. Cockrell Centennial Chair in Engineering and Chairman, Department of Civil Engineering, University of Texas at Austin, Austin, TX 78712-1076

105. Office of Assistant manager for Energy, Research and Development, DOE-ORO, P.O. Box 2001, Oak Ridge, TN 37831

106-107.OSTI, U. S. Department of Energy, P. O. Box 62, Oak Ridge, TN 37831 